\section{A CONSTRUCTION FOR ABSOLUTE VALUES IN POLYNOMIAL RINGS}

BY

\section{SAUNDERS MACLANE}

1. Introduction. An absolute value of a ring is a function $\|b\|$ which has some of the formal properties of the ordinary absolute value. More explicitly, for any $b$ in the ring, $\|b\|$ must be a real number with the properties

$$
\|b c\|=\|b\| \cdot\|c\|, \quad\|b+c\| \leq\|b\|+\|c\| .
$$

If the second inequality holds also in the stronger sense

$$
\|b+c\| \leq \max (\|b\|,\|c\|)
$$

then the value $\|b\|$ is called non-archimedean. The thus delimited nonarchimedean values are of considerable arithmetic interest. They are useful in questions of divisibility and irreducibility and in fact often correspond exactly to the prime ideals of the given ring. This paper is devoted to the explicit construction of non-archimedean values. More specifically, given all such values for the field $R$ of rational numbers, we construct all possible values of the ring $R[x]$ of all polynomials in $x$ with coefficients in $R$.

In treating a non-archimedean value it is convenient to replace $\|a\|$ by a related "exponential" value

$$
V(a)=-\log \|A\|,
$$

with corresponding forms $(\S 2)$ of the formal properties of $V$. The possible nonarchimedean values of the field of rational numbers were determined by Ostrowski (1917): For every prime $p$ there is a $p$-adic exponential value $V_{0}$ in which the value of any rational number is obtained by writing the number as $p^{\alpha}(u / v)$, where $u$ and $v$ are prime to $p$, and setting

$$
V_{0}\left(p^{\alpha}(u / v)\right)=k \alpha,
$$

where $k$ is any fixed positive constant. This value we denote by the symbol $\left[V_{0}(p)=k\right]$. The only other value $V$ is a trivial one, in which $V(a)$ is zero for $a \neq 0$.

On this basis we can determine all possible values in the ring of polynomials with rational coefficients. Any such value $W$ gives a $p$-adic or trivial value $V_{0}(a)=$ $W(a)$ for the rational numbers and a value $\mu=W(x)$ for the variable $x$. These facts alone give a first approximation $V_{1}$ to the value $W$, as follows:

$$
\begin{aligned}
V_{1}\left(a_{n} x^{n}+a_{n-1} x^{n-1}+\cdots+a_{0}\right) \\
\quad=\min \left\{V_{0}\left(a_{n}\right)+n \mu, V_{0}\left(a_{n-1}\right)+(n-1) \mu, \ldots, V_{0}\left(a_{0}\right)\right\} .
\end{aligned}
$$

This $V_{1}$ is actually a value and is never larger than $W$. If $V_{1}$ is not equal to $W$, we choose a $\phi(x)$ of smallest possible degree for which $W(\phi(x))>V_{1}(\phi(x))$. We than define a second approximation $V_{0}(f(x))$ by using* the true value for $\phi(x)$. In this manner we construct successive approximations $V_{1}, V_{2}, V_{3}, \ldots$ which in the limit will give the arbitrary value $W(\S 8)$.

The succession of values $V_{1}, V_{2}, \ldots$ is defined in Part I for polynomials with coefficients in any field $K$. This requires a general method ( $\S \S 4$ and 5 ) of constructing a value $V_{k}$ from a previously obtained value $V_{k-1}$. The value given by the limit of such a sequence needs a special study ( $\$ 7)$. Here, as in $\S \S 8$ and 16 , we assume that every value of the field $K$ is "discrete"; that is, that the real numbers used as values form an isolated point set, as in the case of $p$-adic values.

Part II investigates the structure of the values which have been constructed. The central problem is the construction of the "residue-class field" which arises when polynomials which differ by a polynomial of positive value are put into the same residue-class. For the absolute values constructed in Part I this field is determined by an inductive construction of the homomorphism of polynomials to residueclasses $(\S \S 10-14)$. This homomorphism also yields a more specific description of how our values can be built up $(\S \S 9,13)$. Since a given value $W$ can be represented in many ways by a sequence of approximations $V_{1}, V_{2}, V_{3}, \ldots$, we treat in $\S \S 15$ and 16 the questions as to when two such sequences can give the same ultimate value $W$, and how such a sequence can be put in a normal form.

Among the applications of this construction of absolute values we mention the classification of irreducibility criteria of the Newton Polygon type. The theorem of Eisenstein states that a polynomial

$$
f(x)=x^{n}+a_{n-1} x^{n-1}+a_{n-2} x^{n-2}+\cdots+a_{0}
$$

with integral coefficients $a_{i}$ is irreducible if each coefficient $a_{i}$ is divisible by some fixed prime $p$, while the last term $a_{0}$ is not divisible by $p^{2}$. In terms of the value $V_{1}$ of $(2)$ with $\mu=1 / n$ these hypotheses on $f(x)$ become

$$
V_{1}(f(x))=V_{1}\left(x^{n}\right)=V_{1}\left(a_{0}\right)<V_{1}\left(a_{i} x^{i}\right) \quad(i=1, \ldots, n-1) .
$$

In this form a simple proof of the theorem can be given. The theorems of Königsberger (1895), Dumas (1906), and Ore (1928) are likewise related to the values $V_{1}$. The second stage values $V_{2}$ can be similarly applied to interpret the irreducibility theorems of Schöneman (1846), Bauer (1905), Kürschák (1923), and Ore (1923). By using the general value $V_{k}$ one can obtain a still more extensive irreducibility criterion which includes all these previous theorems (MacLane

\footnotetext{
*Similar "second-stage" values $V_{2}$ appear implicitly in the irreducibility investigations of Ore
} (1923), Kürschák (1923), and Rella (1927). 
(1935)), and which asserts that certain polynomials $f(x)$ with irreducible homomorphic images of sufficiently high degree are themselves irreducible. Our construction for absolute values can also be applied to give a new and complete treatment of the problem of constructing the prime ideal factors of a given rational prime in a given algebraic field.

\section{The CONSTRUCTION OF NON-ARCHIMEDEAN VALUES}

2. Elementary properties of values in rings. A ring* $S$ is said to have a non-archimedean value (for short, a value) $V$ if to every element $a \neq 0$ in $S$ there is assigned a unique real number $V(a)$ with the properties

$$
V(a b)=V(a)+V(b), \quad V(a+b) \geq \min (V(a), V(b)) .
$$

These we call the product and triangle laws respectively. We assume also that 0 is assigned the value $+\infty$, with the following conventions for any finite number $\gamma$ :

$$
\gamma<\infty, \quad \infty+\gamma=\gamma+\infty=\infty+\infty=\infty
$$

Two simple consequences of the product law are

$$
V(1)=V(-1)=0, \quad V(-a)=V(a) .
$$

More important is the strengthened form of the triangle law:

$$
V(a) \neq V(b) \text { implies } V(a+b)=\min (V(a), V(b)) .
$$

For suppose instead that $V(a)>V(b)$ and $V(a+b)>\min (V(a), V(b))$. Then

$$
V(b)=V(a+b-a) \geq \min (V(a+b), V(a))>V(b),
$$

a contradiction.

Since we are using a value analogous to the negative logarithm of the ordinary absolute value, a "small" absolute value will correspond to a "large" value $V$. Hence we say that two ring elements $a$ and $b$ are of the same order of magnitude or equivalent in $V$ - denoted $a \approx_{V} b$ - if and only if

$$
V(a-b)>V(a)
$$

The product and strong triangle laws show that equivalent elements have the same value and that equivalence is a reflexive, symmetric, and transitive relation, provided the supplementary assumption ${ }^{\dagger}$ that $0 \approx_{V} 0$ be made.

\footnotetext{
*Here and in the sequel "ring" means "commutative ring with unit element".

${ }^{\dagger}$ Here and subsequently the element 0 plays an exceptional role.
}

Two equivalences $a \approx_{V} b$ and $c \approx_{V} d$ can be multiplied to give

$$
a c \approx_{V} b d .
$$

An element $b$ is equivalence-divisible in $V$ by $a-$ denoted $a \|_{V} b$ - if and only if there exists a $c$ in $S$ such that

$$
b \approx_{V} c a .
$$

If this is true, it remains true when $a$ or $b$ is replaced by an equivalent element.

The product law implies that a ring $S$ with a value $V$ must be an integral domain. The value $V$ may be extended to the quotient field of $S$ by defining, in accord with the product law,

$$
V\left(\frac{a}{b}\right)=V(a)-V(b)
$$

for any elements $a$ and $b \neq 0$ in $S$. One then obtains the

Theorem 2.1. Let $S$ be an integral domain with quotient field $K$. If $V$ is a value of $S$, then the function defined by (4) is a value of $K$. Conversely, every value of $K$ can be obtained in this way from one and only one value of $S$.

When $S=K$ is a field, the set of all real numbers $V(a)$ for $a \neq 0$ is an additive group $\Gamma$, called the value-group of $V$. If the positive numbers of $\Gamma$ have a positive minimum $\delta>0$, then the value $V$ is said to be discrete. In this case the group $\Gamma$ is cyclic and consists of all multiples of $\delta$. If all elements not 0 have the value 0 , then $V$ is called trivial. Every ring has a trivial value, while the $p$-adic values for the field of rational numbers are examples of discrete values. Values of arithmetic interest are generally discrete.

3. The first stage values. Our problem is this: Given all values of a field $K$; to construct all values for the ring $K[x]$ of all polynomials* in $x$ with coefficients in $K$. By Theorem 2.1, this is equivalent to determining all values in the field $K(x)$ of rational functions of $x$ with coefficients in $K$. No gain in generality would result were a ring $S$ used instead of the field $K$.

As indicated in the introduction, the values for $K[x]$ will be constructed in stages. For the first step, take any value $V_{0}$ for the field $K$ and any real number $\mu$, and then define a corresponding first stage value $V_{1}$ for any polynomial by the equation (2) of $\S 1$. In particular, this gives

$$
V_{1}(x)=\mu, \quad V_{1}(a)=V_{0}(a)
$$

$($ any $a \in K)$.

Hence we use the symbol $\left[V_{0}, V_{1}(x)=\mu\right]$ for the value $V_{1}$.

\footnotetext{
${ }^{*}$ Henceforth all polynomials considered are to have coefficients in $K$, unless otherwise noted.
} 
Theorem 3.1. If $V_{0}$ is a value of $K$ and $\mu$ a real number, the function $\left[V_{0}, V_{1}(x)=\mu\right]$ defined above is a value of $K[x]$.

A particularly simple $V_{1}$ arises when $\mu=0$. On the other hand, if $V_{0}$ is trivial and $\mu<0$, then

$$
V_{1}(a(x))=\mu \operatorname{deg} a(x) .
$$

The symbol $\operatorname{deg} a(x)$ here and in the sequel denotes the degree in $x$ of the polynomial $a(x)$.

4. Augmented values. Our construction now proceeds to build a second stage value on the basis of a first stage one; or, more generally, a $k$ th stage value from one at the stage $k-1$. The process involved can be formulated once for all: Given a value $W$ for $K[x]$; to construct an "augmented" value $V$ by assigning larger values to a certain "key" polynomial $\phi(x)$ and to its equivalence-multiples. The key polynomial $\phi(x)$ must be suitably chosen.

Definition 4.1. A key polynomial $\phi(x) \neq 0$ over a value $W$ of $K[x]$ is one which satisfies the following conditions:

(i) Irreducibility. If a product is equivalence-divisible in $W$ by $\phi(x)$, then one of the factors is equivalence-divisible by $\phi(x)$.

(ii) Minimal degree. Any non-zero polynomial equivalence-divisible in $W$ by $\phi(x)$ has a degree in $x$ not less than the degree of $\phi(x)$.

(iii) The leading coefficient ${ }^{*}$ of $\phi(x)$ is 1 .

This key polynomial is to be assigned a new value

$$
V(\phi(x))=\mu>W(\phi(x)) .
$$

To find the new values of other polynomials, we use expansions in $\phi$; that is, expressions in powers of $\phi(x)$ of the form ${ }^{\dagger}$

$$
f(x)=f_{m}(x) \phi^{m}+f_{m-1}(x) \phi^{m-1}+\cdots+f_{0}(x),
$$

in which each coefficient polynomial $f_{i}(x)$ is either zero or of degree less than the degree of $\phi(x)$. Any polynomial has one and only one such expansion, which may be found by successive division by powers of $\phi$. The new value $V(f(x))$ is computed from the expansion thus:

$$
V\left(f_{m}(x) \phi^{m}+f_{m-1}(x) \phi^{m-1}+\cdots+f_{0}(x)\right)=\min _{i}\left(W\left(f_{i}(x)\right)+i \mu\right) .
$$

Here "min" with subscript $i$ means the smallest quantity of the form $W\left(f_{i}(x)\right)+$ $i \mu$, for $i=0,1, \ldots, m$.

\footnotetext{
${ }^{*}$ This assumption, although unnecessary, will simplify the subsequent work.

${ }^{\dagger}$ We use $\phi$ as an abbreviation for $\phi(x)$, and similarly for other polynomials.
}

Theorem 4.2. If $W$ is a value of $K[x], \phi(x)$ is a key polynomial over $W$ and $\mu$ is a real number satisfying (1), then the function $V$ defined in (3) is also a value of $K[x] . V$ is called an augmented value, and is denoted by

$$
V=[W, V(\phi)=\mu] .
$$

Proof. The product and triangle laws for $V$ must be verified. We first prove the triangle law for a sum $f(x)+g(x)$. Let $f$ and $g$ have the expansions (2) and

$$
g(x)=g_{n}(x) \phi^{n}+g_{n-1}(x) \phi^{n-1}+\cdots+g_{0}(x)
$$

respectively. By adjoining zero coefficients we can make $m=n$. Hence $f+g$ has the expansion

$$
f(x)+g(x)=\sum_{i=0}^{n}\left[f_{i}(x)+g_{i}(x)\right] \phi^{i} .
$$

By the definition of $V$ and the triangle law for $W$,

$$
\begin{aligned}
V(f+g) & =\min _{i}\left(W\left(f_{i}+g_{i}\right)+i \mu\right) \\
& \geq \min _{i}\left(\min \left(W\left(f_{i}\right), W\left(g_{i}\right)\right)+i \mu\right) \\
& \geq \min _{i}\left(W\left(f_{i}\right)+i \mu, W\left(g_{i}\right)+i \mu\right) \\
& =\min \left(\min _{i}\left(W\left(f_{i}\right)+i \mu\right), \min _{i}\left(W\left(g_{i}\right)+i \mu\right)\right),
\end{aligned}
$$$$
V(f+g) \geq \min (V(f), V(g)) .
$$

To prove the product law we will use the quotient-remainder expression for a polynomial $f(x)$,

$$
f(x)=q(x) \phi+r(x)
$$

where $r(x)$ is zero or of degree less than that of $\phi(x)$.

Lemma 4.3. If $\phi$ is a key polynomial over a value $W$ of $K[x]$, and if $f(x) \neq 0$ has the quotient-remainder expression (5), then

$$
\begin{aligned}
W(r(x)) & \geq W(f(x)), \\
W(q(x) \phi) & \geq W(f(x)) .
\end{aligned}
$$

The inequality in (6) holds if and only if $\phi \|_{W} f$.

Proof of Lemma. Were the first conclusion (6) false, then $W(f(x))>W(r(x))$ in (5) and the definition of equivalence would give

$$
r(x) \approx_{W}-q(x) \phi .
$$


Hence $\phi \|_{W} r$ and $r(x) \neq 0$, a contradiction to the minimal property of $\phi$ and the restricted degree of $r(x)$. The second conclusion (7) now follows from (6) by the triangle law.

The third conclusion gives a test for equivalence-divisibility in terms of ordinary division. When $W(r(x))>W(f(x))$, then (5) shows $\phi \|_{W} f$. Conversely, if $\phi \|_{W} f$ then there exist polynomials $h(x)$ and $s(x)$ so that

$$
f(x)=h(x) \phi+s(x), \quad W(s(x))>W(f(x)) .
$$

If now the equality sign in (6) should hold, we would have

$$
r(x)=f(x)-q(x) \phi=(h(x)-q(x)) \phi+s(x),
$$

with

$$
W(s(x))>W(f(x))=W(r(x)),
$$

so that $\phi \|_{W} r$, again a contradiction.

Return to Theorem 4.2 and consider the product law first for a product of two monomial expansions $a(x) \phi^{t}$ and $b(x) \phi^{u}$. Because of the limited degrees of $a(x)$ and $b(x)$, the product $a(x) b(x)$ has an expansion with not more than two terms,

$$
a(x) b(x)=c(x) \phi+d(x) .
$$

Were it the case that $\phi \|_{W} a b$ then the equivalence-irreducibility of $\phi$ (Definition 4.1) would require that either $\phi \|_{W} a$ or $\phi \|_{W} b$, contrary to the minimal property. Hence $\phi \nVdash_{W} a b$. Lemma 4.3 and the triangle axiom then yield

$$
W(c(x) \phi) \geq W(a(x) b(x))=W(d(x)) .
$$

Since the new value of $\phi$ exceeds the old value,

$$
W(c(x))+\mu>W(a(x) b(x))=W(d(x)) .
$$

The product under consideration has by (8) the expansion

$$
\left(a(x) \phi^{t}\right)\left(b(x) \phi^{u}\right)=c(x) \phi^{t+u+1}+d(x) \phi^{t+u} ;
$$

hence the definition of $V$ and the conclusion (9) give

$$
\begin{aligned}
V\left[\left(a(x) \phi^{t}\right)\left(b(x) \phi^{u}\right)\right] & =\min \{W(c(x))+\mu+(t+u) \mu, W(d(x))+(t+u) \mu\} \\
& =W(d(x))+(t+u) \mu \\
& =W(a(x))+t \mu+W(b(x))+u \mu \\
& =V\left(a(x) \phi^{t}\right)+V\left(b(x) \phi^{u}\right) .
\end{aligned}
$$

This is the product law for monomial expansions.

The product law for polynomials $f(x)$ and $g(x)$ with arbitrary expansions (2) and (4) respectively is an immediate consequence. The product $f(x) g(x)$ has an expansion obtained by adding expansions of monomial products; hence

$$
V(f(x) g(x)) \geq V(f(x))+V(g(x)) .
$$

To show that the equality holds, choose $t$ and $u$ as the largest integers with

$$
V\left(f_{t}(x) \phi^{t}\right)=V(f(x)), \quad V\left(g_{u}(x) \phi^{u}\right)=V(g(x))
$$

respectively. The monomial case then shows ${ }^{\ddagger}$ that the expansion of $f(x) g(x)$ has a term $r(x) \phi^{t+u}$ with the value $V(f)+V(g)$. The equality holds in (10), and Theorem 4.2 is established.

5. Properties of augmented values. An augmented value $V$ is never less than the original value $W$. This characteristic property will now be established. As a consequence the method used to compute $V$ can be extended (Theorem 5.2) in a way subsequently useful in $\S 12$.

Theorem 5.1 (Monotonicity). The augmented value $V=[W, V(\phi)=\mu]$ makes

$$
V(f(x)) \geq W(f(x))
$$

for all polynomials $f(x) \neq 0$. The inequality sign holds if and only if $\phi \|_{W} f$. In particular, the equality sign holds whenever the degree of $\phi(x)$ exceeds that of $f(x)$.

Proof. The proof is by induction on the degree $m$ of the expansion of $f(x)$ in $\phi$ (see $\S 4,(2))$. If $m=0$, the definition of $V$ shows $V(f(x))$ and $W(f(x))$ equal. If $m>0$, the quotient-remainder expression

$$
f(x)=q(x) \phi+r(x)
$$

indicates that $q(x)$ has an expansion of degree $m-1$ in $\phi$; hence the induction assumption will be

$$
V(q(x)) \geq W(q(x)) .
$$

The value of the first term on the right of $(1)$, by $\S 4,(1)$, and the quotientremainder Lemma 4.3 , is

$$
V(q(x) \phi) \geq W(q(x))+V(\phi)>W(q(x) \phi) \geq W(f(x)) .
$$

\footnotetext{
$\ddagger$ The details here omitted are given in Rella's proof.
} 
For the second term, the case $m=0$ and Lemma 4.3 imply

$$
V(r(x))=W(r(x)) \geq W(f(x)),
$$

where the inequality holds if and only if $\phi \|_{W} f$. The strong triangle law for $V$ applied to (1) now gives the result (see $\S 2,(2)$ )

Theorem 5.2. If in the expression

$$
a(x)=a_{n}(x) \phi^{n}+a_{n-1}(x) \phi^{n-1}+\cdots+a_{0}(x)
$$

the degrees of the $a_{i}(x)$ are not limited, but $\phi \nVdash_{W} a_{i}$ for $i=0, \ldots, n$ and $a_{i} \neq 0$, then the augmented value $V=[W, V(\phi)=\mu]$ is

$$
V(a(x))=\min _{i}\left[W\left(a_{i}\right)+i \mu\right] \quad(i=0, \ldots, n) .
$$

Proof. A quotient-remainder expression for each coefficient polynomial gives

$$
\begin{array}{rlr}
a_{i}(x) & =q_{i}(x) \phi+r_{i}(x) & (i=0, \ldots, n), \\
a(x) & =\sum_{i=0}^{n} q_{i}(x) \phi^{i+1}+\sum_{i=0}^{n} r_{i}(x) \phi^{i} . &
\end{array}
$$

Lemma 4.3 shows that the second summation has a value

$$
V\left(\sum_{i} r_{i} \phi^{i}\right)=\min _{i}\left[W\left(r_{i}\right)+i \mu\right]=\min _{i}\left[W\left(a_{i}\right)+i \mu\right]
$$

and that the first summation has a larger value

$$
V\left(\sum_{i} q_{i} \phi^{i+1}\right) \geq \min _{i}\left[V\left(q_{i}\right)+V(\phi)+i \mu\right]>\min _{i}\left[W\left(q_{i}\right)+W(\phi)+i \mu\right],
$$

because of the monotonicity. The strong triangle law for the sum of these two summations yields the desired conclusion.

6. Inductive and limit-values. This section classifies the values and valuegroups obtained by successive augmented values.

Definition 6.1. A $k$ th stage inductive value $V_{k}$ is any value of $K[x]$ obtained by a sequence of values $V_{1}, V_{2}, \ldots, V_{k}$, where $V_{1}=\left[V_{0}, V_{1}(x)=\mu_{1}\right]$ is a first stage value $(\S 3)$ and where each $V_{i}$ is obtained by augmenting $V_{i-1}$ :

$$
V_{i}=\left[V_{i-1}, V_{i}\left(\phi_{i}\right)=\mu_{i}\right] \quad(i=2,3, \ldots, k) .
$$

Furthermore, for $i=2, \ldots, k$, the key polynomials $\phi_{i}(x)$ must satisfy: *

$$
\begin{gathered}
\operatorname{deg} \phi_{i}(x) \geq \operatorname{deg} \phi_{i-1}(x) ; \\
\phi_{i}(x) \not_{V_{i-1}} \phi_{i-1}(x) .
\end{gathered}
$$

Here the first key polynomial is understood to be $\phi_{1}(x)=x$.

\footnotetext{
*These conditions involve no loss of generality, but simplify subsequent proofs (see Theorem 6.7 and the end of $\$ 9$ ).
}

The value $V_{k}$ may be conveniently symbolized thus:

(3) $V_{k}=\left[V_{0}, V_{1}\left(\phi_{1}\right)=\mu_{1}, V_{2}\left(\phi_{2}\right)=\mu_{2}, V_{3}\left(\phi_{3}\right)=\mu_{3}, \ldots, V_{k}\left(\phi_{k}\right)=\mu_{k}\right]$.

Given an infinite sequence $V_{1}, V_{2}, \ldots, V_{k}, \ldots$ of such values, we set

$$
V_{\infty}(f(x))=\lim _{k \rightarrow \infty} V_{k}(f(x)) .
$$

The monotonic character of $V_{k}$ indicates that this limit, if not finite, is $+\infty$. $V_{\infty}$ satisfies the product law for values, as can be shown by taking limits in the product law for $V_{k}$. As for the sum $f(x)+g(x)$, note that the triangle law in $V_{k}$ indicates that one of the inequalities

$$
V_{k}(f(x)+g(x)) \geq V_{k}(f(x)), \quad V_{k}(f(x)+g(x)) \geq V_{k}(g(x))
$$

holds for infintely many $k$. One of the conclusions

$$
V_{\infty}(f(x)+g(x)) \geq V_{\infty}(f(x)), \quad V_{\infty}(f(x)+g(x)) \geq V_{\infty}(g(x))
$$

then results, and thence follows the triangle law for $V_{\infty}$. We have

Theorem 6.2. Let $\left\{\phi_{k}(x)\right\}$ and $\left\{\mu_{k}\right\}$ be fixed infinite sequences such that all the functions $V_{k}$ indicated in (3) are inductive values. Then the function $V_{\infty}(f(x))$ defined in (4) is a value of $K[x]$, provided some polynomials not zero be allowed to have the value $+\infty$.

This function $V_{\infty}$ will be called a limit-value. The case when several successive key polynomials have the same degree will often require separate treatment, based on

Lemma 6.3. If in the inductive value $V_{k}$ in (3) the key polynomials $\phi_{t+1}(x)$, $\phi_{t+2}(x), \ldots, \phi_{k}(x)$ all have the same degree, for $t$ with $0 \leq t \leq k-1$, then

$$
\begin{array}{lr}
\text { (i) } V_{t}\left(\phi_{j+1}-\phi_{j}\right)=\mu_{j} & (j=t+1, t+2, \ldots, k-1), \\
\text { (ii) } \mu_{k}>\mu_{k-1}>\cdots>\mu_{t+1}, & \\
\text { (iii) } V_{t}\left(\phi_{k}\right)=V_{t}\left(\phi_{k-1}\right)=\cdots=V_{t}\left(\phi_{t+1}\right) & \quad \text { (if } t>0)
\end{array}
$$

Proof. Let $j$ range from $t+1$ to $k-1$, and set

$$
s_{j}(x)=\phi_{j+1}(x)-\phi_{j}(x) .
$$

Since both $\phi$ 's have the first coefficient 1 , the degree of $s_{j}(x)$ is less than that of $\phi_{j}(x)$. Therefore, by Theorem 5.1,

$$
V_{t}\left(s_{j}(x)\right)=V_{t+1}\left(s_{j}(x)\right)=\cdots=V_{k}\left(s_{j}(x)\right) .
$$


If the first conclusion were false for some $j$, we would have

$$
V_{t}\left(s_{j}(x)\right)=V_{j}\left(\phi_{j+1}-\phi_{j}\right)>\mu_{j}=V_{j}\left(\phi_{j}\right)
$$

for the other inequality is impossible by Lemma 4.3. This would give

$$
\phi_{j+1} \approx_{V_{j}} \phi_{j}
$$

a contradiction of assumption (2). The conclusion (i) is thus established. Coupled with the monotonicity and the triangle axiom for (5), it gives the second conclusion, for

$$
\mu_{j+1}=V_{j+1}\left(\phi_{j+1}\right)>V_{j}\left(\phi_{j+1}\right) \geq \min \left\{V_{j}\left(\phi_{j}\right), V_{t}\left(s_{j}\right)\right\}=\mu_{j} .
$$

For similar reasons, assuming now that $t>0$,

$$
V_{t}\left(s_{j}(x)\right)=\mu_{j}=V_{j}\left(\phi_{j}\right)>V_{j-1}\left(\phi_{j}\right) \geq V_{t}\left(\phi_{j}\right)
$$

The strong triangle axiom for $V_{t}$ in (5) then yields conclusion (iii),

$$
V_{t}\left(\phi_{j+1}\right)=\min \left\{V_{t}\left(s_{j}(x)\right), V_{t}\left(\phi_{j}\right)\right\}=V_{t}\left(\phi_{j}\right)
$$

An interesting consequence of this lemma is the invariance of the values assigned to the key polynomials.

Theorem 6.4. If the ith stage of the inductive value $V_{k}$ in (3) uses a key polynomial $\phi_{i}$ with an assigned value $\mu_{i}$, then

$$
V_{k}\left(\phi_{i}(x)\right)=V_{i}\left(\phi_{i}(x)\right)=\mu_{i}
$$

For this conclusion follows directly from Theorem 5.1 if the degree of $\phi_{i+1}(x)$, and hence that of every subsequent key polynomial, exceeds the degree of $\phi_{i}(x)$. The only case remaining is that of Lemma 6.3 , with $t=i-1$. But, by (5),

$$
\phi_{i}=\phi_{k}-s_{k-1}(x)-s_{k-2}(x)-\cdots-s_{i}(x) .
$$

The terms on the right have by the preceding lemma the $V_{k}$ values $\mu_{k}$, $\mu_{k-1}, \ldots, \mu_{i}$ respectively, so that the conclusion follows by the strong triangle law. Both this theorem and Lemma 6.3 hold equally well for limit-values.

The monotonic property of inductive values can be sharpened thus:

Theorem 6.5. Let a limit or inductive value be built up by the inductive values $V_{1}, V_{2}, \ldots$ Then, for any fixed polynomial $f(x) \neq 0$, either

$$
V_{k+1}(f(x))>V_{k}(f(x))
$$$$
(k=1,2, \ldots),
$$

or else there is an $i \geq 1$ such that

$$
\begin{aligned}
V_{1}(f(x))<V_{2}(f(x))<\cdots< & V_{i-1}(f(x)) \\
& =V_{i}(f(x))=V_{i+1}(f(x))=V_{i+2}(f(x))=\cdots .
\end{aligned}
$$

In the latter case there is an $r(x)$ of degree less than that of $\phi_{i+1}$ with

$$
f(x) \approx_{V_{k}} r(x) \quad(k=i+1, i+2, \ldots) .
$$

Suppose, contrary to the first alternative, that for some $i$

$$
V_{i+1}(f(x))=V_{i}(f(x))
$$

Then the quotient-remainder expression

$$
f(x)=q(x) \phi_{i+1}+r(x)
$$

must by Theorem 5.1 and Lemma 4.3 have $V_{i}(r)=V_{i}(f)$. Hence, for any $k \geq i+1$,

$$
V_{k}(f-r) \geq V_{i+1}(f-r)=V_{i+1}\left(q \phi_{i+1}\right)>V_{i}\left(q \phi_{i+1}\right) \geq V_{i}(f)=V_{i}(r)=V_{k}(r) .
$$

Therefore $f(x) \approx_{V_{k}} r(x)$ and

$$
V_{k}(f)=V_{k}(r)=V_{i}(r)=V_{i}(f),
$$

so that $V_{i}(f(x))$ is constant for $k \geq i$, which is the second alternative.

An inductive value $V_{k}$ of $K[x]$ gives by Theorem 2.1 a value for the field $K(x)$ of rational functions. This value has by $\S 2$ a value-group $\Gamma_{k}$, which we call the value-group associated with $V_{k}$. It may be determined in the following way:

Theorem 6.6. The value $V_{k}$ in (3) has a value-group $\Gamma_{k}$ consisting of all real numbers of the form

$$
\nu+m_{1} \mu_{1}+m_{2} \mu_{2}+\cdots+m_{k} \mu_{k}
$$

where the $m_{i}$ are integers and $\nu$ is an element of the value-group of the original value $V_{0}$.

That every number of $\Gamma_{k}$ must be of this form follows by induction from the definition of the augmented value $V_{k}$. Conversely, any number of this form is by Theorem 6.4 the value in $V_{k}$ of the rational function

$$
b x^{m_{1}} \phi_{2}^{m_{2}} \cdots \phi_{k}^{m_{k}}
$$

where $b$ is a constant in $K$ with the value $\nu$.

For a more precise description, designate a real number $\mu$ as commensurable with an additive group of numbers whenever some integral multiple of $\mu$ lies in the group. Then 
Theorem 6.7. In an inductive value $V_{k}$ from (3) every assigned value $\mu_{i}$, except perhaps $\mu_{k}$, is commensurable with the value-group $\Gamma_{i-1}$ of the preceding value (the case $i=1$ included).

Proof. Consider the expansion in $\phi_{i}$ of the next key,

$$
\phi_{i+1}(x)=f_{m}(x) \phi_{i}^{m}+f_{m-1}(x) \phi_{i}^{m-1}+\cdots+f_{0}(x) .
$$

If $\mu_{i}$ is not commensurable with $\Gamma_{i-1}$, no two terms here can have the same value in $V_{i}$. Only one term, say the $j$ th, has the minimum value, and

$$
\phi_{i+1}(x) \approx_{V_{i}} f_{j}(x) \phi_{i}^{j}
$$

By the irreducibility of $\phi_{i+1}$ at least one of the conditions

$$
\begin{aligned}
& \phi_{i+1} \|_{V_{i}} f_{j}(x), \\
& \phi_{i+1} \|_{V_{i}} \phi_{i},
\end{aligned}
$$

must hold. Because of the minimal property of $\phi_{i+1}$ the first possibility (6) contradicts the assumption (1) of Definition 6.1. For the same reasons the second possibility (7) implies that $\phi_{i+1}$ and $\phi_{i}$ have the same degree, while

$$
s(x)=\phi_{i+1}(x)-\phi_{i}(x)
$$

has a smaller degree. Because of (7), Lemma 4.3 applied to $V_{i}$ and the key polynomial $\phi_{i+1}$ shows $V_{i}(s(x))>V_{i}\left(\phi_{i+1}(x)\right)$. Hence

$$
\phi_{i}(x) \approx_{V_{i}} \phi_{i+1}(x),
$$

a contradiction of assumption (2). There can be no next key $\phi_{i+1}$.

7. Constant degree limit-values. A limit-value $V_{\infty}$ for polynomials does not give a value for all rational functions if some of the polynomials have the value $+\infty$. Hence the problem: When is $V_{\infty}$ finite; that is, when is $V_{\infty}(f(x))$ finite for all $f(x) \neq 0$ ? We obtain an answer in the discrete case.

If the key polynomials $\phi_{k}(x)$ increase indefinitely in degree, then $V_{k}(f(x))$ is by Theorem 5.1 ultimately constant for fixed $f(x)$ and $V_{\infty}$ is finite. A different situation arises if the degrees of $\phi_{k}(x)$ are then all equal to some $M$ for $k$ sufficiently large. For an example of such a constant degree limit-value, start with the $p$-adic value $\left[V_{0}(3)=1\right]$ for the rational field ( see $\left.\S 1,(1)\right)$ and set

$$
\begin{aligned}
& V_{1}=\left[V_{0}, V_{1}(x)=1\right] \\
& V_{k}=\left[V_{k-1}, V_{k}\left(x+2 p+p^{2}+p^{3}+\cdots+p^{k-1}\right)=k\right] \quad(k=2,3, \ldots) .
\end{aligned}
$$

This gives a limit-value of constant degree 1 . Since

$$
\frac{p}{2}=2 p+p^{2}+p^{3}+\cdots+p^{k-1}-\frac{p^{k}}{2} \quad(k>1, p=3)
$$

holds by the usual methods for $p$-adic numbers, we find

$$
V_{\infty}\left(x+\frac{p}{2}\right)=\lim _{k \rightarrow \infty} V_{k}\left(\left(x+2 p+p^{2}+p^{3}+\cdots+p^{k-1}\right)-\frac{p^{k}}{2}\right)=\lim _{k \rightarrow \infty} k=\infty .
$$

Hence this $V_{\infty}$ is not finite.

This use of $p$-adic numbers suggests the general notion of a perfect ring. In any ring $S$ with a value $V$, a sequence $\left\{a_{n}\right\}$ is a Cauchy sequence if $V\left(a_{n}-a_{m}\right)$ approaches $\infty$ with $n$ and $m$. If every Cauchy sequence has a $V$-limit $b$ such that $V\left(a_{n}-b\right)$ approaches $\infty$ with $n$, the ring $S$ is said to be perfect. Any ring can be embedded in a perfect ring by the usual procedure of adjoining limits of Cauchy sequences.

Theorem 7.1 (Finiteness criterion). Let $V_{\infty}$ be a limit-value with key polynomials $\phi_{k}(x)$ of constant degree $M$ for $k>t>0$. Extend the ring $K[x]$ with the value $V_{t}$ to be a perfect ring $S^{*}$. Assume that all values of $K$ are discrete. Then $\left\{\phi_{k}\right\}$ is a Cauchy sequence in $V_{i}$ and has a limit $\phi$ in $S^{*}$. Furthermore $V_{\infty}$ is finite if and only if there is no $g(x) \neq 0$ in $K[x]$ divisible in $S^{*}$ by the limit $\phi$.

For $V_{\infty}$ the symbolism of Theorem 6.2 may be used. Since $\phi_{t+1}, \phi_{t+2}, \ldots$ all have the same degree $M$, the conclusions of Lemma 6.3 on constant degree values are applicable. Each number $\mu_{i}$ is by Theorem 6.7 commensurable with the valuegroup $\Gamma_{i-1}$ of $V_{i-1}$. Our assumption shows the original value-group $\Gamma_{0}$ of $V_{0}$ to be discrete, hence, by Theorem 6.6 and by induction, the group $\Gamma_{t}$ is discrete. But Lemma 6.3 gives

$$
\mu_{i}=V_{i}\left(\phi_{i+1}-\phi_{i}\right) \in \Gamma_{i}
$$

hence $\Gamma_{i}=\Gamma_{t}$ for $i>t$. This lemma also shows the sequence $\left\{\mu_{i}\right\}$ to be monotone increasing for $i>t$; it lies in the discrete set $\Gamma_{t}$, hence

$$
\lim _{i \rightarrow \infty} \mu_{i}=\infty
$$

The strong triangle law combined with (1) then proves

$$
V_{t}\left(\phi_{i+j}-\phi_{i}\right)=V_{t}\left(\sum_{k=i}^{i+j-1}\left(\phi_{k+1}-\phi_{k}\right)\right)=\min _{k}\left\{\mu_{k}\right\}=\mu_{i} .
$$

Therefore, by (2), $\left\{\phi_{i}\right\}$ is a Cauchy sequence with a limit $\phi$ in $S^{*}$. This $\phi$ need not be a polynomial, but, by conclusion (iii) of Lemma $6.3, \phi \neq 0$. 
Now consider the necessary condition for finiteness. If $g(x) \neq 0$ is divisible by $\phi$ in $S^{*}$, then

$$
g(x)=h \phi,
$$

where $h$ is the $V_{i}$-limit of a Cauchy sequence $\left\{h_{i}(x)\right\}$ from $K[x]$. The usual argument for the convergence of a product shows

$$
\lim _{i \rightarrow \infty} V_{t}\left(g(x)-h_{i}(x) \phi_{i}(x)\right)=\infty .
$$

By the triangle axiom and the monotonic property for $i>t$,

(4) $V_{i}(g) \geq \min \left\{V_{i}\left(h_{i} \phi_{i}\right), V_{i}\left(g-h_{i} \phi_{i}\right)\right\} \geq \min \left\{V_{t}\left(h_{i}\right)+\mu_{i}, V_{t}\left(g-h_{i} \phi_{i}\right)\right\}$.

But $\left\{h_{i}(x)\right\}$ is a convergent sequence in $V_{i}$ with a limit not zero, so that, as is well known, $V_{t}\left(h_{i}\right)$ is ultimately constant. Consequently $(2),(3)$, and (4) prove

$$
V_{\infty}(g(x))=\lim _{i \rightarrow \infty} V_{i}(g(x))=\infty
$$

so that $V_{\infty}$ is not a finite limit-value.

Conversely, suppose that $V_{\infty}$ is not finite. Then (5) holds for some $g(x) \neq 0$. If $g(x)$ has the quotient-remainder expressions $q_{i}(x) \phi_{i}+r_{i}(x)$, then, by Theorem 5.1 and by Lemma 4.3 ,

$$
V_{i}\left(g-q_{i} \phi_{i}\right)=V_{i}\left(r_{i}\right)=V_{i-1}\left(r_{i}\right) \geq V_{i-1}(g(x)) \rightarrow \infty \quad(i>t) .
$$

Thus the sequence $\left\{q_{i} \phi_{i}\right\}$ converges in $V_{i}$ to the limit $g(x) \neq 0$. Since $\left\{\phi_{i}\right\}$ already converges to the limit $\phi \neq 0$, the standard argument for the limit of a quotient $\left(q_{i} \phi_{i}\right) / \phi_{i}$ shows that $\left\{q_{i}\right\}$ must converge in $V_{i}$ to some limit $q$ in $S^{*}$, such that

$$
f(x)=q \phi .
$$

Hence $\phi$ is a factor of $f(x)$ in $S^{*}$, as asserted.

8. Completeness. We have the following theorem.

Theorem 8.1. If every value of the field $K$ is discrete, then every non-archimedean value $W$ of the ring $K[x]$ can be represented either as an inductive or as a limit-value.

Given $W$, we shall construct by stages a corresponding inductive value $V_{k}$ with the following three properties (notation as in $\S 6,(3)$ ):

$$
\begin{array}{cc}
W(f(x)) \geq V_{k}(f(x)) & (\text { for all } f(x)), \\
\operatorname{deg} f(x)<\operatorname{deg} \phi_{k} \text { implies } W(f(x))=V_{k}(f(x)), & \\
W\left(\phi_{i}(x)\right)=V_{k}\left(\phi_{i}(x)\right)=\mu_{i} \quad(i=1,2, \ldots, k) .
\end{array}
$$

The initial value $V_{1}$ is defined by

$$
\mu_{1}=W(x), \quad V_{0}(a)=W(a)
$$

$($ any $a \in K)$;

the triangle axiom for $W$ and the definition of $V_{1}$ in $\S 1,(2)$, then show that conditions (1), (2), and (3) hold for $k=1$.

Suppose now that an inductive value $V_{k}$ with these three properties has already been constructed, and that the equality in (1) does not always hold. As a prospective key polynomial, choose a $\psi(x)$ of smallest possible degree with the property

$$
W(\psi(x))>V_{k}(\psi(x)) .
$$

Multiplication with some constant gives $\psi(x)$ the first coefficient 1. Furthermore the two statements

$$
\begin{gathered}
W(f(x))>V_{k}(f(x)), \\
\psi(x) \|_{V_{k}} f(x),
\end{gathered}
$$

are logically equivalent. For if (5) is given, and if $f(x)$ has the quotient-remainder expression $q(x) \psi+r(x)$, then

$$
V_{k}(q \psi-f)=W(q \psi-f) \geq \min \{W(q \psi), W(f)\}>\min \left\{V_{k}(q \psi), V_{k}(f)\right\},
$$

because of (2), the minimum degree choice of $\psi$ and the induction assumption (1) for $q(x)$. Hence the strong triangle law shows $f \approx_{V_{k}} q \psi$, which is the conclusion (6). Conversely, if (6) holds there exist polynomials $h(x)$ and $s(x)$ with

$$
f(x)=h(x) \psi+s(x), \quad V_{k}(s(x))>V_{k}(f(x))=V_{k}(h(x) \psi) .
$$

Then, because of the induction assumption (1),

$$
\begin{aligned}
W(f) \geq \min \{ & W(h \psi), W(s)\} \\
& \geq \min \left\{V_{k}(h)+W(\psi), V_{k}(s)\right\}>V_{k}(h)+V_{k}(\psi)=V_{k}(f),
\end{aligned}
$$

which gives conclusion (5). The equivalence of (5) and (6) is established.

From the equivalence one readily shows that $\psi(x)$ satisfies the Definition 4.1 of a key polynomial over the value $V_{k}$. Finally we can assign $\psi(x)=\phi_{k+1}$ the new value

$$
\mu_{k+1}=W(\psi)>V_{k}(\psi)
$$

satisfying the proper inequality, and then construct the augmented value $V_{k+1}=$ $\left[V_{k}, V_{k+1}\left(\phi_{k+1}\right)=\mu_{k+1}\right]$. This will be an inductive value if only conditions (1) 
and (2) of Definition 6.1 hold. By the choice of $\phi_{k+1}=\psi$ and the induction assumption (2), $\phi_{k}(x)$ cannot exceed $\phi_{k+1}(x)$ in degree, therefore condition (1) of $\S 6$ is true. Condition (2) of $\S 6$ could only be false if $\phi_{k+1} \approx_{V_{k}} \phi_{k}$; in other words, only if

$$
V_{k}\left(\phi_{k}-\phi_{k+1}\right)>V_{k}\left(\phi_{k}\right)=V_{k}\left(\phi_{k+1}\right) .
$$

By (2), (3), and the choice of $\psi$ in (4) this would entail

$$
\begin{aligned}
W\left(\phi_{k}\right) \geq \min \left\{W\left(\phi_{k+1}\right)\right. & \left., W\left(\phi_{k}-\phi_{k+1}\right)\right\} \\
& >\min \left\{V_{k}\left(\phi_{k+1}\right), V_{k}\left(\phi_{k}\right)\right\}=V_{k}\left(\phi_{k}\right)=W\left(\phi_{k}\right),
\end{aligned}
$$

a contradiction which establishes the desired condition.

The inductive value $V_{k+1}$ thus constructed satisfies the analogues of the desired conditions (1), (2), and (3). The latter two are consequences of the definitions in (4) and (7), while (1) follows from the definition (see $\S 4,(3))$ of the augmented value $V_{k+1}$ by the triangle axiom for $W$ :

$$
W\left(\sum_{i=0}^{m} f_{i}(x) \psi^{i}\right) \geq \min _{i}\left(W\left(f_{i}(x)\right)+i \mu_{k+1}\right)=V_{k+1}\left(\sum_{i=0}^{m} f_{i}(x) \psi^{i}\right) .
$$

The inductive construction of the value $V_{k}$ associated with $W$ is complete.

This process either will ultimately yield an inductive value $V_{k}$ equal to $W$ or will give an infinite sequence of inductive values with a limit-value $V_{\infty}$ such that

$$
\left.W(f(x)) \geq V_{\infty}(f(x))=\lim _{k \rightarrow \infty} V_{k}(f(x)) \quad \text { (for all } f(x)\right) .
$$

In the discrete case the first inequality sign never occurs. For suppose instead that it did hold for some $f(x)$; then since $\left\{V_{k}(f)\right\}$ is monotone non-decreasing,

$$
W(f(x))>V_{k}(f(x)) \quad(k=1,2, \ldots) .
$$

The equivalence of (5) and (6) then implies that $\phi_{k+1}(x) \|_{V_{k}} f(x)$. Hence the monotonicity Theorem 5.1 shows

$$
V_{k+1}(f(x))>V_{k}(f(x))
$$$$
(k=1,2, \ldots) \text {. }
$$

This cannot hold if the degrees of the key polynomials $\phi_{k}(x)$ increase indefinitely, so that we have the case where $\phi_{k}(x)$ has the fixed degree $M$ for $k>t$, as in Theorem 7.1. The monotonic increasing sequence $\left\{V_{k}(f(x))\right\}$ consists of numbers all from the discrete group $\Gamma_{t}$, with the result

$$
W(f(x)) \geq V_{\infty}(f(x))=\lim _{k \rightarrow \infty} V_{k}(f(x))=\infty .
$$

This can occur only for $f(x)=0$, a trivial case. Accordingly, $W=V_{\infty}$, and the completeness theorem is established.

\section{The STRUCTURE OF INDUCTIVE VALUES}

9. Properties of key polynomials. To apply the preceding construction of values to any particular case it is necessary to know what polynomials can be used as key polynomials. This question is not constructively answered by the definition in $\S 4$. Part of this question will be answered at once (Theorem 9.4); the rest after the structure of the inductive values $V_{k}$ has been more explicitly formulated. We first show that certain polynomials act like "equivalence-units":

Lemma 9.1. If $V_{k}$ is an inductive value with $k>1$, then for every polynomial $b(x)$ with $V_{k}(b(x))=V_{k-1}(b(x))$ there is a polynomial $b^{\prime}(x)$ with

$$
b^{\prime}(x) b(x) \approx_{V_{k}} 1, \quad V_{k}\left(b^{\prime}(x)\right)=V_{k-1}\left(b^{\prime}(x)\right) .
$$

The hypothesis on $b(x)$ implies that $b(x)$ is not divisible by the last key polynomial $\phi_{k}(x)$. Since $\phi_{k}$ is certainly irreducible in the ordinary sense, there are polynomials $b^{\prime}(x)$ and $c(x)$ with

$$
b^{\prime}(x) b(x)+c(x) \phi_{k}(x)=1, \quad \operatorname{deg} b^{\prime}(x)<\operatorname{deg} \phi_{k}(x) .
$$

By Theorem 5.1, $V_{k}\left(b^{\prime}\right)=V_{k-1}\left(b^{\prime}\right)$. The transition from $V_{k-1}$ to $V_{k}$ increases the value of $c \phi_{k}$, but leaves unchanged the values of $b^{\prime} b$ and 1 in this equation. Hence $b^{\prime} b \approx_{V_{k}} 1$, as in $(1)$.

Lemma 9.2. In any inductive $V_{k}$, the last key polynomial $\phi_{k}$ is equivalenceirreducible in $V_{k}$; a polynomial $g(x)$ with $\phi_{k} \nVdash_{v_{k}} g(x)$ has a value $V_{k}(g)$ in $\Gamma_{k-1}$.

Proof. If a polynomial $f(x)$ has the expansion

then $\phi_{k} \|_{V_{k}} f(x)$ if and only if $V_{k}\left(f_{0}\right)>V_{k}(f)$. For if $V_{k}\left(f_{0}\right)>V_{k}(f)$, then $f-f_{0}$ is a polynomial equivalent to $f$ with a factor $\phi_{k}$. Conversely, if $f \approx_{V_{k}} h(x) \phi_{k}$, then the last term $f_{0}$ of the expansion for $f$ is obtained from $f-h \phi_{k}$, where $V_{k}\left(f-h \phi_{k}\right)>V(f)$, so that $V_{k}\left(f_{0}\right)>V_{k}(f)$. In particular, if $\phi_{k} \nVdash_{v_{k}} f$ then $V_{k}(f)=V_{k}\left(f_{0}\right)=V_{k-1}\left(f_{0}\right) \in \Gamma_{k-1}$, as asserted.

This criterion shows $\phi_{k}$ equivalence-irreducible in $V_{k}$. For suppose instead that $\phi_{k} \|_{V_{k}} f g$, although neither factor is so divisible. Then the criterion gives $V_{k}\left(f_{0}\right)=$ $V_{k}(f), V_{k}\left(g_{0}\right)=V_{k}(g)$, where $g_{0}(x)$ is the last term in the expansion for $g$. The last term in the expansion for $f g$ is the remainder $r_{0}(x)$ obtained by dividing $f_{0} g_{0}$ by $\phi_{k}$; but since $\phi_{k} \nVdash_{v_{k-1}} f_{0} g_{0}$, Lemma 4.3 shows

$$
V_{k}\left(r_{0}\right)=V_{k-1}\left(r_{0}\right)=V_{k-1}\left(f_{0} g_{0}\right)=V_{k}\left(f_{0}\right)+V_{k}\left(g_{0}\right)=V_{k}(f g) \text {. }
$$

This means that $\phi_{k} \nVdash_{v_{k}} f g$, a contradiction proving the lemma. 
An inductive value $V_{k}$ will be called commensurable if the value $\mu_{k}$ assigned the last key polynomial is commensurable with the previous value-group $\Gamma_{k-1}$ (cf. Theorem 6.7). There is then a smallest positive integer $\tau_{k}$ such that $\tau_{k} \mu_{k}$ is in $\Gamma_{k-1}$. For each $t \leq k$ there is a similar $\tau_{t}$ :

$$
\tau_{t} \text { is the smallest integer such that } \tau_{t} \mu_{t} \in \Gamma_{t-1} \text {. }
$$

We will subsequently need polynomials with any given values:

Lemma 9.3. If $V_{k}$ is a commensurable inductive value, then for any real number $\lambda$ in the value-group $\Gamma_{k}$ of $V_{k}$ there is a polynomial $R_{\lambda}=R_{\lambda}(x)$ with value $\lambda$ in $V_{k}$ and in every value $V_{k+1}$ obtained by augmenting $V_{k}$.

Proof. As in Theorem 6.6, $\lambda$ has the form

$$
\lambda=\nu+m_{1} \mu_{1}+m_{2} \mu_{2}+\cdots+m_{k} \mu_{k}, \quad \nu \in \Gamma_{0} .
$$

Each integer $m_{k}$ may be made non-negative by adding to $m_{i} \mu_{i}$ and subtracting from $\nu$ a sufficiently large term $q \mu_{i}$, so chosen that $q \mu_{i} \in \Gamma_{0}$ (e.g., choose $q \equiv 0$ $\left.\left(\bmod \tau_{1}, \tau_{2}, \ldots, \tau_{i}\right)\right)$. If then $a$ is a constant of value $\nu$,

$$
R_{\lambda}=R_{\lambda}(x)=a x^{m_{1}} \phi_{2}^{m_{2}} \phi_{3}^{m_{3}} \cdots \phi_{k}^{m_{k}}, \quad V_{k}\left(R_{\lambda}\right)=\lambda, \quad m_{k} \geq 0,
$$

is the required polynomial. In any augmented value $V_{k+1}, R_{\lambda}$ has value $\lambda$ by Theorem 6.4 .

Theorem 9.4. A polynomial $f(x)$ is a key polynomial for an inductive value over $V_{k}$ if and only if the following conditions hold:

(i) the expansion (2) has a last term with $V_{k}(f)=V_{k}\left(f_{0}\right)$;

(ii) the expansion has a first term $f_{n}(x) \phi_{k}^{n}$ with $f_{n}(x)=1, V_{k}\left(\phi_{k}^{n}\right)=V_{k}(f)$, and $n \equiv 0\left(\bmod \tau_{k}\right)$;

(iii) $f(x)$ is equivalence-irreducible in $V_{k}$.

Proof. Condition (i) means, as in the proof of Lemma 9.2, that $\phi_{k}(x) \nVdash_{v_{k}} f(x)$. Assume first that $f(x)$ is a key. Condition (iii) is necessary by definition. Were (i) false, then $V_{k}(f)<V_{k}\left(f_{0}\right)$, so that $f \approx_{V_{k}}\left(f-f_{0}\right)$, while $(2)$ shows $f-f_{0}=q(x) \phi_{k}$ for a $q(x)$ of degree less than $f(x)$. Thus $f \approx_{V_{k}} q \phi_{k}$. Since $f$ is a key, this leads to a contradiction much as in the proof of Theorem 6.7. The assumption $V_{k}\left(f_{0}\right) \neq V_{k}(f)$ is false.

Since $f(x)$ is minimal (Definition 4.1) it has no equivalence-multiples of degree less than itself. Hence $f_{n}(x)$ is a constant in $K$, for otherwise $k>1$, and Lemma 9.1 supplies a $b^{\prime}(x)$ with $b^{\prime}(x) f_{n}(x) \approx_{V_{k}} 1$. The product $b^{\prime}(x) f(x)$ formed from $(2)$ and modified by replacing the first coefficient by 1 and by reducing the other coefficients modulo $\phi_{k}$ is then an equivalence-multiple of $f(x)$. Its degree is $n \operatorname{deg} \phi_{k}$, and is less than that of $f(x)$ unless $f_{n}(x) \in K$. As the leading coefficient of $f$ must be $1, f_{n}(x)=1$ follows, as in (ii). Certainly $V_{k}\left(\phi_{k}^{n}\right)=V_{k}(f)$ is necessary, for otherwise $f-\phi_{k}^{n}$ is an equivalent polynomial of smaller degree. Thus

$$
V_{k}\left(\phi_{k}^{n}\right)=V_{k}(f)=V_{k}\left(f_{0}\right)=V_{k-1}\left(f_{0}\right) \in \Gamma_{k-1},
$$

so that $n \equiv 0\left(\bmod \tau_{k}\right)$ by $(3)$. This establishes the necessity of (ii).

Conversely, if $f(x)$ satisfies (i), (ii), and (iii), it has first coefficient 1 and is minimal, because any equivalence-multiple of $f(x)$ must be of degree at least $n$ in $\phi_{k}$ (cf. the proof of Theorem 4.2). The remaining restrictions of Definition 6.1 are readily verified, so that $f(x)$ is in fact a key polynomial.

10. Residue-class fields. The structure of a $\operatorname{ring} S$ with a value $V$ involves the corresponding value-ring $S^{+}$, which consists of all elements $a$ of $S$ with $V(a) \geq 0$ (these elements are the so-called "integers" of $S$ ). A congruence for integers can be defined thus

$$
a \equiv b(\bmod V) \text { if and only if } V(a-b)>0 .
$$

All elements of $S^{+}$congruent to a given $b$ form a residue-class; these classes together yield as usual the residue-class ring of $V$ in $S$. This ring can also be considered as the residue-class ring $S^{+} / P$, where $P$, the set of all elements of $S^{+}$ with positive value, is a prime ideal in $S^{+}$. If $S$ is a field, then $S^{+} / P$ is also a field, the residue-class field of $V$ in $S$. The structure of $V$ depends essentially on this residue-class field. For the $p$-adic value $V_{0}$ of the rational numbers (see $\S 1$, (1)) this field is simply the field of integers modulo $p$. Our problem is the determination of the residue-class field for any discrete inductive value.

If the residue-class of each integer $a$ be denoted by $\llbracket a \rrbracket$, then $H: a \mapsto \llbracket a \rrbracket$ is a homomorphism of $S^{+}$to the residue-class ring $\Delta=S^{+} / P$, so that $H$ has the following properties:

I. $H$ is a many-one correspondence between $S^{+}$and $\Delta$;

II. $H$ leaves sums and products unchanged; i.e., for $V(a) \geq 0$ and $V(b) \geq 0$ :

$$
\llbracket a+b \rrbracket=\llbracket a \rrbracket+\llbracket b \rrbracket ; \quad \llbracket a b \rrbracket=\llbracket a \rrbracket \llbracket b \rrbracket .
$$

III. If $V(a) \geq 0$, then $\llbracket a \rrbracket=\llbracket 0 \rrbracket$ if and only if $V(a)>0$.

By II, the last condition means that $H$ carries congruent elements and only congruent elements into the same residue-class. 
For an inductive value $V_{k}$ we denote the residue-class rings thus, for $t=1$, $2, \ldots, k$ :

$$
\Lambda_{t} \text { is the residue-class field of } V_{t} \text { in } K(x) \text {; }
$$

$H_{t}$ is the homomorphism from $K(x)^{+}$to $\Lambda_{t}$;

$\Delta_{t}$ is the residue-class ring of $V_{t}$ in $K[x]$.

But $f(x)$ and $g(x)$ are congruent as polynomials $\left(\bmod V_{t}\right.$ ) if and only if they are congruent as rational functions $\left(\bmod V_{t}\right)$. Hence each residue-class of $\Delta_{t}$ is contained in a residue-class of $\Lambda_{t}$, and no two residue-classes of $\Delta_{t}$ are contained in the same class of $\Lambda_{t}$. Addition and multiplication of classes are defined as addition and multiplication on elements in the classes, and hence are the same in $\Delta_{t}$ as in $\Lambda_{t}$. Therefore $\Delta_{t}$ is isomorphic to a subring of $\Lambda_{t}$. Since isomorphism does not alter the structure of a ring, we will replace $\Delta_{t}$ henceforth by the isomorphic subring of $\Lambda_{t}$. Then the $H_{t}$ of (4) is also the homomorphism from $K[x]^{+}$to $\Delta_{t}$.

The correspondence $H_{t}$ for rational functions is usually determined by the $H_{t}$ for polynomials. For if $f(x) / g(x) \neq 0$ is a rational function with non-negative value and if $V_{t}$ is commensurable, there is by Lemma 9.3 a polynomial $g^{b_{t}}(x)$ with $V_{t}\left(g^{b_{t}}\right)=-V_{t}(g)$, and by $(2)$

$$
\llbracket \frac{f(x)}{g(x)} \rrbracket_{t}=\llbracket \frac{g^{b_{t}} f}{g^{b_{t}} g} \rrbracket_{t}=\frac{\llbracket g^{b_{t}} f \rrbracket_{t}}{\llbracket g^{b_{t}} g \rrbracket_{t}} .
$$

Both $\llbracket g^{b_{t}} f \rrbracket_{t}$ and $\llbracket g^{b_{t}} g \rrbracket_{t}$ are residue-classes of polynomials, while $\llbracket g^{b_{t}} g \rrbracket_{t} \neq \llbracket 0 \rrbracket_{t}$ by Property III. We have proved

Lemma 10.1. For a commensurable $V_{k}$, the residue-class field $\Lambda_{k}$ of $K(x)$ is the quotient-field of the residue-class ring $\Delta_{k}$ of $K[x]$.

Theorem 10.2. For a commensurable first stage inductive value

$$
V_{1}=\left[V_{0}, V_{1}(x)=\mu_{1}\right],
$$

the residue-class ring $\Delta_{1}$ is isomorphic to the ring $F_{0}[y]$ of all polynomials in a variable $y$ with coefficients in $F_{0}$, the residue-class field of the value $V_{0}$ for $K$.

Proof. There is given a homomorphism $H_{0}$ from the ring $K^{+}$of all $V_{0}$-integers $b$ in $K$ to the residue-class field $F_{0}$. Each residue-class $\llbracket b \rrbracket_{1}$ of $\Delta_{1}$ contains the residue-class $\llbracket b \rrbracket_{0}$ of $F_{0}$, and this correspondence $\llbracket b \rrbracket_{1} \longleftrightarrow \llbracket b \rrbracket_{0}$ is an isomorphism between $F_{0}$ and the set of those classes of $\Delta_{1}$ containing elements of $K$. We will identify $F_{0}$ with this isomorphic subfield of $\Delta_{1}$; then $\Delta_{1}$ is an extension of $F_{0}$ and $\llbracket b \rrbracket_{1}=\llbracket b \rrbracket_{0}$ for all $b$ in $K^{+}$.
Any monomial $b x^{n}$ of value zero has $V_{0}(b)=-n V_{1}(x)=-n \mu_{1}$, so that the exponent $n$ is a multiple of the integer $\tau_{1}$, defined in $\S 9,(3)$. Any $f(x)$ with $V_{1}(f) \geq 0$ thus has the form

$$
f(x) \equiv b_{m} x^{m \tau_{1}}+b_{m-1} x^{(m-1) \tau_{1}}+\cdots+b_{1} x^{\tau_{1}}+b_{0} \quad\left(\bmod V_{1}\right)
$$

after terms of positive value are omitted. If $e$ is a constant in $K$ of value $V_{0}(e)=$ $\tau_{1} \mu_{1}$, each term $b_{j} x^{j \tau_{1}}$ may be rewritten as a product $\left(b_{j} e^{j}\right)\left(e^{-j} x^{j \tau_{1}}\right)$ of two factors of value 0 . The application of the homomorphism $H_{1}$ then yields

$$
\llbracket f(x) \rrbracket_{1}=\sum_{j=0}^{m} \llbracket b_{j} e^{j} \rrbracket_{0} y^{j} ; \quad y=\llbracket e^{-1} x^{\tau_{1}} \rrbracket_{1} .
$$

With $y$ so defined, any $\llbracket f \rrbracket_{1}$ in $\Delta_{1}$ becomes a polynomial in $y$ with coefficients $\llbracket b_{j} e^{j} \rrbracket_{0}$ in $F_{0}$, so that the residue-class ring $\Delta_{1}$ is contained in $F_{0}[y]$. Since $y \in \Delta_{1}$ and $\Delta_{1}$ is a ring, $\Delta_{1}=F_{0}[y]$. The element $y$ is transcendental (i.e., a variable) over $F_{0}$, for otherwise it would satisfy an algebraic relationship $\alpha(y)=\llbracket 0 \rrbracket_{1}$, where

$$
\alpha(y)=\alpha_{m} y^{m}+\alpha_{m-1} y^{m-1}+\cdots+\alpha_{0} ; \quad \alpha_{m} \neq \llbracket 0 \rrbracket_{1}, \quad \alpha_{j} \in F_{0} .
$$

Then the residue-class $\alpha(y)$ contains the polynomial

$$
f(x)=a_{m} e^{-m} x^{m \tau_{1}}+a_{m-1} e^{-m+1} x^{(m-1) \tau_{1}}+\cdots+a_{0},
$$

where each $a_{j}$ is a constant with $\llbracket a_{j} \rrbracket_{0}=\alpha_{j}$. Then $V_{1}(f) \geq 0$ and $\llbracket f \rrbracket_{1}=$ $\alpha(y)=\llbracket 0 \rrbracket_{1}$, so that, by Property III of $H_{1}, V_{1}(f)>0$. But $\alpha_{m} \neq \llbracket 0 \rrbracket_{1}$, so that $V_{0}\left(a_{m}\right)=0$ and $V_{1}\left(a_{m} e^{-m} x^{m \tau_{1}}\right)=V_{1}(f)=0$, a contradiction. The theorem is established. We note also that (7) enables us to calculate the residue-class of any given $f(x)$.

11. Conditions for equivalence-irreducibility. If $\phi_{k+1}$ is a key polynomial over a value $V_{k}$ then $\phi_{k} \nVdash_{v_{k}} \phi_{k+1}$ (Theorem 9.4, condition (i)). For any $f(x)$ with this property, questions of equivalence-divisibility can be handled as follows:

Lemma 11.1. In a commensurable $V_{k}$ let $f(x)$ be a polynomial with $\phi_{k} \nVdash_{v_{k}} f$, and choose a polynomial $f^{b_{k}}(x)$ so that $V_{k-1}\left(f^{b_{k}}\right)=V_{k}\left(f^{b_{k}}\right)=-V_{k}(f)$. Then a polynomial $g(x)$ with $V_{k}(g)=0$ satisfies $f \|_{V_{k}} g$ if and only if $\llbracket g \rrbracket_{k}$ is divisible by $\llbracket f^{b_{k}} f \rrbracket_{k}$ in the residue-class ring $\Delta_{k}$.

By Lemma 9.2, $V_{k}(f)$ is in $\Gamma_{k-1}$, so Lemma 9.3 yields the $f^{b_{k}}$ desired, and $\llbracket f^{b_{k}} f \rrbracket_{k} \neq \llbracket 0 \rrbracket_{k}$. Suppose first that $\llbracket g \rrbracket_{k}$ (which is not $\llbracket 0 \rrbracket_{k}$ by Property III of $\left.H_{k}\right)$ is divisible by $\llbracket f^{b_{k}} f \rrbracket_{k}$. Then $\llbracket g \rrbracket_{k}=\alpha \llbracket f^{b_{k}} f \rrbracket_{k}$ for some residue-class $\alpha=\llbracket h(x) \rrbracket_{k} \neq \llbracket 0 \rrbracket_{k}$ in $\Delta_{k}$, and

$$
\llbracket g \rrbracket_{k}=\alpha \llbracket f^{b_{k}} f \rrbracket_{k}=\llbracket h \rrbracket_{k} \llbracket f^{b_{k}} f \rrbracket_{k}=\llbracket h f^{b_{k}} f \rrbracket_{k} .
$$


Thus $g$ and $h f^{b_{k}} f$ have the same residue-class, $V_{k}\left(g-h f^{b_{k}} f\right)>0=V_{k}(g)$, so $g \approx_{V_{k}} h f^{b_{k}} f$ and $f \|_{V_{k}} g$, as asserted.

Conversely, if $f \|_{V_{k}} g$, then $g \approx_{V_{k}} h f \approx_{V_{k}} h f^{\sharp_{k}} f^{b_{k}} f$, where $f^{\sharp_{k}}(x)$ is a polynomial chosen as in Lemma 9.1 so that $f^{b_{k}} f^{\sharp_{k}} \approx_{V_{k}} 1$. But $f^{b_{k}} f, g$, and hence $h f^{\sharp_{k}}$ have value 0 , so that

$$
g \approx_{V_{k}} h f^{\sharp_{k}} f^{b_{k}} f ; \quad \llbracket g \rrbracket_{k}=\llbracket h f^{\sharp_{k}} \rrbracket_{k} \llbracket f^{b_{k}} f \rrbracket_{k},
$$

which shows $\llbracket g \rrbracket_{k}$ divisible by $\llbracket f^{b_{k}} f \rrbracket_{k}$.

Lemma 11.2. For $f$ and $f^{b_{k}}$ as in Lemma 11.1, $f(x)$ is equivalence-irreducible in $V_{k}$ if and only if every product in $\Delta_{k}$ divisible by $\llbracket f^{b_{k}} f \rrbracket_{k}$ has ${ }^{*}$ a factor divisible by $\llbracket f^{b_{k}} f \rrbracket_{k}$ in $\Delta_{k}$.

Proof. Suppose first that $f$ is equivalence-irreducible and that $\llbracket g \rrbracket_{k} \llbracket h \rrbracket_{k}=\llbracket g h \rrbracket_{k}$ is a multiple of $\llbracket f^{b_{k}} f \rrbracket_{k}$. As we can assume $V_{k}(g)=V_{k}(h)=0$, the previous lemma shows the product $g h$ equivalence-divisible by the equivalence-irreducible $f$, so that one of the factors is so divisible. By Lemma 11.1 this means that $\llbracket g \rrbracket_{k}$ or $\llbracket h \rrbracket_{k}$ is a multiple of $\llbracket f^{b_{k}} f \rrbracket_{k}$, as asserted in the lemma.

Conversely, suppose that every product $\llbracket g \rrbracket_{k} \llbracket h \rrbracket_{k}$ divisible by $\llbracket f^{b_{k}} f \rrbracket_{k}$ has a factor so divisible, and consider a product $a(x) b(x)$ with $f \|_{V_{k}} a b$, so that $a b \approx_{V_{k}} c(x) f$ for some $c$. Write $a(x) \approx_{V_{k}} g(x) \phi_{k}^{d}$ and $b(x) \approx_{V_{k}} h(x) \phi_{k}^{e}$, where the powers $d$ and $e$ are chosen so large that $\phi_{k} \nVdash_{v_{k}} g$ and $\phi_{k} \nVdash_{v_{k}} h$. Then $V_{k}(g)$ and $V_{k}(h)$ are by Lemma 9.2 in $\Gamma_{k-1}$, so that there are polynomials $g^{b_{k}}(x)$ and $h^{b_{k}}(x)$ with $V_{k}\left(g^{b_{k}} g\right)=V_{k}\left(h^{b_{k}} h\right)=0$. Then

$$
g^{b_{k}} h^{b_{k}} a b \approx_{V_{k}}\left(g^{b_{k}} g\right)\left(h^{b_{k}} h\right) \phi_{k}^{d+e} \approx_{V_{k}} g^{b_{k}} h^{b_{k}} c f .
$$

But $\phi_{k} \nVdash_{v_{k}} f$, while $\phi_{k}$ is equivalence-irreducible (Lemma 9.2), so

$$
\phi_{k}^{d+e} \|_{V_{k}} g^{b_{k}} h^{b_{k}} c .
$$

Removal of the factor $\phi_{k}^{d+e}$ gives $f \|_{V_{k}}\left(g^{b_{k}} g\right)\left(h^{b_{k}} h\right)$, so that as in the previous lemma $\llbracket g^{b_{k}} g \rrbracket_{k} \llbracket h^{b_{k}} h \rrbracket_{k}$ is divisible by $\llbracket f^{b_{k}} f \rrbracket_{k}$. One of the factors, say $\llbracket g^{b_{k}} g \rrbracket_{k}$, is then divisible by $\llbracket f^{b_{k}} f \rrbracket_{k}$, and (Lemma 11.1) $f \|_{V_{k}} g^{b_{k}} g$. But $a \approx_{V_{k}} g^{\sharp_{k}}\left(g^{b_{k}} g\right) \phi_{k}^{d}$, where $g^{\sharp_{k}}$ is chosen so that $g^{\sharp_{k}} g^{b_{k}} \approx_{V_{k}} 1$. Hence $f \|_{V_{k}} a(x)$, and $f$ is equivalenceirreducible.

${ }^{*}$ That is, the principal ideal $\left(\llbracket f^{b_{k}} f \rrbracket_{k}\right)$ is a prime ideal in $\Delta_{k}$.
12. Residue-class rings for commensurable values. We have

Theorem 12.1. If $V_{k}$ is a commensurable inductive value of $K[x]$, given as in $\S 6,(3)$, and if the original value $V_{0}$ of $K$ has a residue-class field $F_{0}$, then there is a sequence of fields $F_{1}=F_{0}, F_{2}, F_{3}, \ldots, F_{k}$, each an algebraic extension of the preceding, such that for any $t=1, \ldots, k$ the $V_{t}$-residue-class ring of $K[x]$ is (isomorphic to) the ring $F_{t}[y]$ of polynomials in a variable $y$ with coefficients in $F_{t}$. For $t>1$ the degree $m_{t}$ of $F_{t}$ is determined by (cf. $\S 9,(3)$ )

$$
m_{t} \tau_{t-1} \operatorname{deg} \phi_{t-1}=\operatorname{deg} \phi_{t} ; \quad m_{t}=\operatorname{deg}\left[F_{t}: F_{t-1}\right] .
$$

By Lemma 10.1 we can then conclude at once

Corollary 12.2. $F_{t}(y)$ is the $V_{t}$-residue-class field of $K(x)$.

Proof of Theorem. The case $t=1$ of this theorem is known (Theorem 10.2); hence we use induction, and assume the theorem true for $V_{t}$. It is convenient to omit the subscript $t+1$ (but not the subscript $t$ ) and to write $V, \phi, H, \tau$, etc., for $V_{t+1}, \phi_{t+1}, H_{t+1}, \tau_{t+1}$, etc. By the monotonic character of $V$ (Theorem 5.1) polynomials $f(x)$ and $g(x)$ with $V_{t}(f) \geq 0$ and $V_{t}(g) \geq 0$ are congruent $\bmod V_{t}$ only if they are congruent $\bmod V$. Each residue-class mod $V_{t}$ is thus contained in a residue-class $\bmod V$, and this gives a homomorphism between $\Delta_{t}=F_{t}[y]$ and a subring $F$ of the residue-class ring $\Delta(\mathrm{cf} . \S 10,(5))$, where $F=F_{t+1}$ is composed of all residue-classes mod $V$ containing an $f(x)$ with $V_{t}(f) \geq 0$. Polynomials $f$ and $g$ incongruent $\bmod V_{t}$ become congruent $\bmod V$ if and only if $\phi_{t+1} \|_{V_{t}} f-g$ (Theorem 5.1). This means that $\llbracket f \rrbracket_{t}-\llbracket g \rrbracket_{t}$ is divisible by the polynomial

(1) $\quad \psi_{t+1}(y)=\psi(y)=\llbracket \phi^{b_{t}} \phi \rrbracket_{t}$

$$
\left(V_{t-1}\left(\phi^{b_{t}}\right)=V_{t}\left(\phi^{b_{t}}\right)=-V_{t}(\phi), \phi^{b_{t}}=\phi_{t+1}^{b_{t}}(x)\right),
$$

constructed as in Lemma 11.1. Since not all polynomials are equivalence-divisible by $\phi$ in $V_{t}, \psi(y)$ is not a constant in $F_{t}$, while Lemma 11.2 shows $\psi(y)$ an irreducible polynomial in $F_{t}[y]$. In the above homomorphism between $F_{t}[y]$ and $F$ the mutiples of $\psi(y)$ in $F_{t}[y]$ are the elements corresponding to $\llbracket 0 \rrbracket$, so that $F$ is isomorphic to the ring of polynomials $F_{t}[y]$ modulo $\psi(y)$, or, alternatively, to the field obtained by adjoining to $F_{t}$ a root $\theta$ of $\psi(y)$. We identify $F$ with this isomorphic field:

$$
F=F_{t+1}=F_{t}(\theta) ; \quad \psi(\theta)=0
$$$$
\left(\theta=\theta_{t+1}\right) .
$$

Then the residue-class $\llbracket f \rrbracket_{t}$, when reduced modulo $\psi(y)$, will be identical to the residue-class $\llbracket f \rrbracket$; that is,

$$
V_{t}(f(x)) \geq 0 \text { implies } \llbracket f \rrbracket=\left.\llbracket f \rrbracket_{t}\right|_{y=\theta} .
$$


A monomial expansion $a(x) \phi^{n}$ of value 0 must have $n$ a multiple of $\tau$ (cf. $\S 9$,

$(3))$. Hence any $f(x)$ with $V(f) \geq 0$ has the form

(4) $f(x) \equiv f_{m}(x) \phi^{m \tau}+f_{m-1}(x) \phi^{(m-1) \tau}+\cdots+f_{0}(x)(\bmod V)$,

$$
\operatorname{deg} f_{i}<\operatorname{deg} \phi
$$

Since $V\left(\phi^{\tau}\right) \in \Gamma_{t}$, there are by Lemmas 9.1 and 9.3 polynomials $\phi^{\tau \sharp}(x)$ and $\phi^{\tau b}(x)$ such that

$$
\begin{aligned}
V_{t}\left(\phi^{\tau \sharp}\right)=V\left(\phi^{\tau \sharp}\right)=V\left(\phi^{\tau}\right), \quad \phi^{\tau \sharp} \phi^{\tau b} \equiv & 1(\bmod V), \\
& V_{t}\left(\phi^{\tau b}\right)=V\left(\phi^{\tau b}\right)=-V\left(\phi^{\tau}\right) .
\end{aligned}
$$

The terms $f_{i} \phi^{i \tau}$ in (4) can be rewritten as products $\left(f_{j} \phi^{\tau \sharp j}\right)\left(\phi^{j \tau}\left(\phi^{\tau \sharp}\right)^{-j}\right)$, where $V_{t}\left(f_{j} \phi^{\tau \sharp j}\right) \geq 0$ and $V\left(\phi^{j \tau}\left(\phi^{\tau \sharp}\right)^{-j}\right)=0$, the former because $V(f) \geq 0$. The application of $H$, with (3), then proves

$$
\llbracket f(x) \rrbracket=\left.\sum_{j=0}^{m} \llbracket f_{j}(x) \phi^{\tau \sharp j} \rrbracket_{t}\right|_{y=\theta} y_{1}^{j} ; \quad y_{1}=\llbracket \phi^{\tau}\left(\phi^{\tau \sharp}\right)^{-1} \rrbracket .
$$

This shows that every $\llbracket f \rrbracket$ in the residue-class ring $\Delta$ is also in $F\left[y_{1}\right]$, while by $(5)$, $y_{1}=\llbracket \phi^{\tau}\left(\phi^{\tau \sharp}\right)^{-1} \rrbracket=\llbracket \phi^{\tau} \phi^{\tau b} \rrbracket$ is a residue-class of a polynomial, hence is in $\Delta$. Consequently, $F\left[y_{1}\right]=\Delta$, as asserted in the theorem.

The element $y_{1}$ is transcendental over $F$; for suppose instead that $y_{1}$ satisfied an algebraic relation $\alpha\left(y_{1}\right)=\llbracket 0 \rrbracket$, with

$$
\alpha\left(y_{1}\right)=\alpha_{m} y_{1}^{m}+\alpha_{m-1} y_{1}^{m-1}+\cdots+\alpha_{0} ; \quad \alpha_{m} \neq \llbracket 0 \rrbracket, \quad \alpha_{j} \in F .
$$

By the original (italicized) definition of $F$ each residue-class $\alpha_{j}$ of $F$ contains a polynomial $b_{j}(x)$ with $V_{t}\left(b_{j}\right) \geq 0$, so that $\llbracket b_{j} \rrbracket=\alpha_{j}$. Then

$$
f(x)=\sum_{j=0}^{m} b_{j}(x) \phi^{\tau b j} \phi^{j \tau} \equiv \sum_{j=0}^{m} b_{j}(x)\left(\phi^{\tau \sharp}\right)^{-j} \phi^{j \tau}
$$

is a polynomial of non-negative value which has $\llbracket f \rrbracket=\alpha\left(y_{1}\right)=\llbracket 0 \rrbracket$. By Property III of $H, V(f)>0$. On the other hand $V(f)$ must equal 0 , for $\llbracket b_{m} \rrbracket=\alpha_{m} \neq \llbracket 0 \rrbracket$ gives $V\left(b_{m}\right)=0$ and $V(f) \leq V\left(b_{m}\left(\phi^{\tau \sharp}\right)^{-m} \phi^{m \tau}\right)=0$, by Theorem 5.2. This contradiction shows $y_{1}$ a variable over $F$.

The formula (6) enables us to calculate $\llbracket f(x) \rrbracket$ effectively for any $f(x)$ given in (4), provided only that $V_{t}\left(f_{j} \phi^{\tau \sharp j}\right) \geq 0$ for all $j$.

It remains to determine the degree of the field $F$ over $F_{t}$, which by $(2)$ is the degree of $\psi(y)$. The key $\phi$ has by Theorem 9.4 an expansion of the form

$$
\phi=\phi_{t}^{m \tau_{t}}+\sum_{i=0}^{m \tau_{t}-1} a_{i}(x) \phi_{t}^{i}, \quad V_{t}(\phi)=V_{t}\left(\phi_{t}^{m \tau_{t}}\right)=V_{t}\left(a_{0}\right)
$$

If $t>1, \psi=\llbracket \phi^{b_{t}} \phi \rrbracket_{t}$ can be computed by the analog of (6) for the preceding stage (with $t$ in (6) replaced by $t-1$ ), for the coefficients $\phi^{b_{t}} a_{i}$ must by the choice of $\phi^{b_{t}}$ have $V_{t-1}\left(\phi^{b_{t}} a_{i}\right) \geq 0$. This calculation shows $\psi(y)$ to be a polynomial in $y$ with a first term $\llbracket \phi^{b_{t}} \phi_{t}^{\tau_{t} \sharp_{t} m} \rrbracket_{t} y^{m}$ arising from the first term of (7). But

$$
V_{t}\left(\phi^{b_{t}} \phi_{t}^{\tau_{t} \sharp_{t} m}\right)=V_{t}\left(\phi^{b_{t}}\right)+V_{t}\left(\phi_{t}^{\tau_{t} \sharp_{t} m}\right)=-V_{t}(\phi)+V_{t}\left(\phi_{t}^{m \tau_{t}}\right)=0,
$$

so that the coefficient of $y^{m}$ is not $\llbracket 0 \rrbracket$. The polynomial $\psi$ has degree $m$, and by $(7)$

$$
m \tau_{t} \operatorname{deg} \phi_{t}=\operatorname{deg} \phi, \quad m=\operatorname{deg} \psi=\operatorname{deg}\left[F: F_{t}\right],
$$

as asserted* in Theorem 12.1. This theorem has now been demonstrated.

13. Conditions for key polynomials. In the criterion of Theorem 9.4 for a key polynomial the condition (iii) of equivalence-irreducibility can now be replaced by the condition of Lemma 11.2,

$$
\llbracket f^{b_{k}} f \rrbracket_{k} \text { is an irreducible polynomial in } F_{k}[y] \text {. }
$$

This yields a final explicit description of key polynomials. A partial converse is possible:

Theorem 13.1. In a given $V_{k}$, let $\psi(y) \neq y$ be a polynomial ${ }^{\dagger}$ of degree $m>0$, irreducible in $F_{k}[y]$ and with first coefficient 1 . Then there is one and, except for equivalent polynomials in $V_{k}$, only one $\phi(x)$ which is a key polynomial and which has $\llbracket \phi_{k}^{\tau_{k} b_{k} m} \phi \rrbracket_{k}=\psi(y)$.

Proof. There is a polynomial $f(x)$ with the residue-class $\psi$, so that $\llbracket f \rrbracket_{k}=\psi$ and $V_{k}(f)=0$. If we multiply $f$ by $\phi_{k}^{\tau_{k} \sharp_{k} m}$ (chosen as in $\S 12,(5)$ ) and in the expansion of the resulting product drop all terms not of minimum value and then replace the leading coefficient of $\phi_{k}$ by 1 , we obtain a polynomial $\phi(x)$ with the value $V_{k}\left(\phi_{k}^{\tau_{k} \sharp_{k} m}\right)$. Then

$$
\llbracket \phi_{k}^{\tau_{k} b_{k} m} \phi \rrbracket_{k}=\llbracket \phi_{k}^{\tau_{k} b_{k} m} \phi_{k}^{\tau_{k} \sharp_{k} m} f \rrbracket_{k}=\llbracket f \rrbracket_{k}=\psi(y) .
$$

Furthermore $\phi$ can be shown to satisfy the remaining conditions (i) and (ii) of Theorem 9.4, hence $\phi$ is a key polynomial. The uniqueness is readily established.

\footnotetext{
*The proof given holds for $t>1$, but may be simplified for the case $t=1$.

${ }^{\dagger}$ The assumption $\psi(y) \neq y$ is needed, for the condition $V_{k}(f)=V_{k}\left(f_{0}\right)$ in Theorem 9.4
} implies $\llbracket f^{b_{k}} f \rrbracket_{k} \neq y$. 
Since $\llbracket f^{b_{k}} f \rrbracket_{k}$ can be effectively constructed by $\S 12,(6)$, the problem of testing whether a given $f(x)$ is a key polynomial is reduced to that of testing the image $\llbracket f^{b_{k}} f \rrbracket_{k}$ of $f(x)$ for irreducibility in $F_{k}[y]$. If $K$ is the field of rationals, then $F_{k}$ is a finite field and the latter problem is completely solvable. This result can be used to construct examples for inductive values of any stage and for limitvalues of both constant degree ${ }^{\ddagger}$ and increasing degree types. The construction of constant degree values may be simplified by deducing from Theorem 9.4 the following partial converse of Lemma 6.3:

Corollary 13.2. In $V_{k}$ let $s(x)$ be a polynomial of degree less than that of $\phi_{k}(x)$ and with $V_{k}(s(x))=V_{k}\left(\phi_{k}\right)$. Then $\phi_{k}(x)+s(x)$ is a key polynomial for an inductive value over $V_{k}$.

14. Special cases of homomorphism. The residue-class fields can be similarly found for finite discrete limit-values and for inductive values where the value for $K$ is trivial $(\S 2)$ or where the last assigned value $\mu_{k}$ is incommensurable $(\S 6)$.

Theorem 14.1. Let $V_{\infty}$ be a limit-value constructed as in Theorem 6.1 from a sequence of values $V_{0}, V_{1}, V_{2}, \ldots$, and satisfying one of the conditions:

(a) the degrees of the keys $\phi_{k}$ are not bounded as $k \rightarrow \infty$;

(b) $V_{\infty}$ is finite and discrete, and $\operatorname{deg} \phi_{k}=M$ for all $k>t$.

The fields $F_{k}$ of Theorem 12.1 yield a (possibly infinite) extension field $F_{\infty}=$ $F_{0}+F_{1}+F_{2}+\cdots$ which is isomorphic to the $V_{\infty}$ residue-class field both for $K[x]$ and for $K(x)$. In case (b), $F_{\infty}=F_{t+1}$.

Let $H_{k}$ be the homomorphism of $K[x]$ to the $V_{k}$ residue-class ring $F_{k}[y]$ and $H_{\infty}$ the homomorphism of $K[x]$ to the $V_{\infty}$ residue-class ring $\Delta_{\infty}$. Then

$$
V_{k-1}(f(x)) \geq 0 \text { implies that } \llbracket f \rrbracket_{k+j}=\llbracket f \rrbracket_{k} \in F_{k} \quad(j=1,2, \ldots) .
$$

For, according to $\S 12,(3)$,

$$
\llbracket f \rrbracket_{k}=\left.\llbracket f \rrbracket_{k-1}\right|_{y=\theta_{k}}, \quad \llbracket f \rrbracket_{k+1}=\left.\llbracket f \rrbracket_{k}\right|_{y=\theta_{k+1}},
$$

which indicates that $\llbracket f \rrbracket_{k}$ is a constant free of $y$ in $F_{k}$, and that $\llbracket f \rrbracket_{k+1}$ must equal $\llbracket f \rrbracket_{k}$, and so on.

In case (a) there is for every $f(x)$ a $k$ so large that $\operatorname{deg} \phi_{k}>\operatorname{deg} f$, so that $V_{\infty}(f)=V_{k-1}(f)$, as in Theorem 5.1. If $V_{\infty}(f) \geq 0$, then, by $(1), \llbracket f \rrbracket_{k+j}=\llbracket f \rrbracket_{k}$ is a constant in $F_{k}$ independent of $j$.

\footnotetext{
${ }^{\ddagger}$ By using a transcendental $p$-adic number the finiteness condition of $\S 7$ can be satisfied.
}

The correspondence

$$
\llbracket f \rrbracket_{\infty} \longleftrightarrow \llbracket f \rrbracket_{k}, \quad \text { for } k \text { with } \llbracket f \rrbracket_{k}=\llbracket f \rrbracket_{k+1}=\llbracket f \rrbracket_{k+2}=\cdots,
$$

carries each element of $\Delta_{\infty}$ into an element of $F_{\infty}$. Every element $\alpha$ of $F_{\infty}$ is used, for, by the definition of $F_{\infty}, \alpha$ is in some $F_{k}$ so that $\alpha$ has the form $\llbracket f \rrbracket_{k}$, and $\llbracket f \rrbracket_{k+j}=\llbracket f \rrbracket_{k}$ as in (1), whence $\alpha$ corresponds in (2) to $\llbracket f \rrbracket_{k}$. The correspondence (2) is one-to-one, for elements are congruent $\bmod V_{\infty}$ if and only if they are congruent modulo some $V_{k}$. Finally, (2) is an isomorphism, making $F_{\infty} \cong \Delta_{\infty}$, as asserted. The residue-class field of $K(x)$ is, by the argument of Lemmas 9.3 and 10.1, just the quotient field of $F_{\infty}$, and must then be $F_{\infty}$ itself.

In the case (b), the degrees of the extensions $F_{k+1}: F_{k}$ as determined in Theorem 12.1 are all 1 for $k>t$. Hence $F_{k}=F_{t+1}$. Because $V_{\infty}$ is finite $(\S 7)$ and discrete, Theorem 6.5 yields for any $f(x)$ with $V_{\infty}(f) \geq 0$ an $i \geq t$ so large that $V_{\infty}(f)=V_{i}(f) \geq 0$. Then $\llbracket f \rrbracket_{k}$ is again ultimately constant, and (2) gives the isomorphism as before.

Theorem 14.2. For an incommensurable inductive value $V_{k}$ of $K(x)$ the field $F_{k}$ determined from $F_{k-1}$ and $\phi_{k}$ exactly as in $\S 12$ is the $V_{k}$ residue-class field of both $K[x]$ and $K(x)$.

Proof. Since no non-zero multiple of $\mu_{k}=V_{k}\left(\phi_{k}\right)$ lies in $\Gamma_{k-1}$, no two terms in a $\phi_{k}$-expansion can have the same value in $V_{k}$. Hence any polynomial is equivalent to a monomial expansion, and every rational function has by Lemma 9.1 the form

$$
f(x) / g(x) \approx_{V_{k}} c(x) \phi_{k}^{m}, \quad V_{k}(c)=V_{k-1}(c) .
$$

If $f / g$ has value 0 , then $m=0, V_{k-1}(c) \geq 0$, and $\llbracket f / g \rrbracket_{k}=\llbracket c \rrbracket_{k}$. But $F_{k}$ is defined in $\S 12$, italics (or, for $k=1$, in $\S 10$ ) as all residue-classes $\llbracket h \rrbracket_{k}$ with $V_{k-1}(h) \geq 0$. In this case every residue-class has this form, so that $F_{k}$ is as asserted the whole residue-class field, either for $K[x]$ or for $K(x)$.

In particular, over the trivial value $V_{0}(\S 2)$ of $K$ the only non-trivial inductive values are

$$
\begin{aligned}
& V_{1}=\left[V_{0}, V_{1}(x)=\mu_{1}\right], \quad \quad \mu_{1} \neq 0 ; \\
& V_{2}=\left[V_{0}, V_{1}(x)=0, V_{2}(\phi)=\mu_{2}\right], \quad \mu_{2}>0, \phi(x) \text { irreducible. }
\end{aligned}
$$

Both are incommensurable (no multiple of $\mu_{2}$ lies in the group $\Gamma_{0}$, which contains only 0). Furthermore, the residue-class field of $K$ for the trivial $V_{0}$ is $K$ itself. Hence the residue-class field for $V_{1}$ is $K$ and for $V_{2}$ is $K(\theta)$, where $\theta$ is a root of $\phi(x)$. 
15. Equality conditions for values. An inductive value is essentially a representation; the same value of $K[x]$ could easily have several such representations. This section and the next one will formulate necessary and sufficient conditions for the equality of two inductive or limit-values. In this connection two values $V$ and $W$ of a ring $S$ will be called equal if an only if

$$
V(a)=W(a)
$$

(all $a \in S$ )

In this section we consider the case when each key polynomial $\phi_{k}$ exceeds the preceding $\phi_{k-1}$ in degree - a case which can often be made to apply by omitting any $\phi_{k-1}$ without the above property.

Lemma 15.1. If an inductive value

$$
V_{k}=\left[V_{k-2}, V_{k-1}\left(\phi_{k-1}\right)=\mu_{k-1}, V_{k}\left(\phi_{k}\right)=\mu_{k}\right]
$$

has two key polynomials $\phi_{k-1}(x)$ and $\phi_{k}(x)$ of the same degree, then

$$
W=\left[V_{k-2}, W\left(\phi_{k}\right)=\mu_{k}\right]
$$

is an inductive value equal to $V_{k}$.

We first prove $W$ an inductive value. Since $\phi_{k}$ exceeds $\phi_{k}-\phi_{k-1}$ in degree, the constant-degree Lemma 6.3 shows that

(2) $V_{k-2}\left(\phi_{k}-\phi_{k-1}\right)=V_{k-1}\left(\phi_{k}-\phi_{k-1}\right)=\mu_{k-1}, \quad \mu_{k}>\mu_{k-1}>V_{k-2}\left(\phi_{k-1}\right)$.

A combination of these two results proves

$$
\phi_{k} \approx_{V_{k-2}} \phi_{k-1}
$$

Thus $\phi_{k}$ and $\phi_{k-1}$ have the same equivalence-divisibility properties in $V_{k-2}$, and so $\phi_{k}$, like $\phi_{k-1}$, is a key polynomial over $V_{k-2}$. By (2) and (3) the value $\mu_{k}>V_{k-2}\left(\phi_{k}\right)$ assigned to $\phi_{k}$ is sufficiently large. Therefore $W$ is inductive, for conditions (1) and (2) of Definition 6.1 hold trivially. The definition of augmented values applied to the usual expansion (e.g., $\S 9,(2))$ of any $f(x)$ in powers of $\phi_{k}$ gives

$$
V_{k}(f(x))=\min _{i}\left\{V_{k-1}\left(f_{i}(x)\right)+i \mu_{k}\right\}, W(f(x))=\min _{i}\left\{V_{k-2}\left(f_{i}(x)\right)+i \mu_{k}\right\} .
$$

The corresponding terms $V_{k-1}\left(f_{i}\right)$ and $V_{k-2}\left(f_{i}\right)$ are equal by Theorem 5.1 , for each $V_{i}(x)$ has a degree less than that of $\phi_{k}$ or of $\phi_{k-1}$. Therefore $V_{k}=W$. Successive applications of this lemma give

Theorem 15.2. Any inductive value is equal to an inductive value in which $\left\{\operatorname{deg} \phi_{k}\right\}$ is a monotone increasing sequence. A similar representation holds for any limit-value not of constant-degree type.
For values in this particular form we can obtain necessary and sufficient conditions for equality.

Theorem 15.3. If the two inductive values

$$
\begin{aligned}
V_{s} & =\left[\begin{array}{ll}
V_{0}, \quad V_{1}(x) & =\mu_{1}, V_{2}\left(\phi_{2}\right)=\mu_{2}, \ldots, V_{s}\left(\phi_{s}\right)=\mu_{s}
\end{array}\right] \\
W_{t} & =\left[\begin{array}{ll}
W_{0}, W_{1}(x) & =\nu_{1}, W_{2}\left(\psi_{2}\right)=\nu_{2}, \ldots, W_{s}\left(\psi_{t}\right)=\nu_{t}
\end{array}\right]
\end{aligned}
$$

both have a monotone character such that

$$
1<\operatorname{deg} \phi_{2}<\cdots<\operatorname{deg} \phi_{s}, \quad 1<\operatorname{deg} \psi_{2}<\cdots<\operatorname{deg} \psi_{t},
$$

then $V_{s}=W_{t}$ holds if and only if

$$
\begin{aligned}
& \text { (i) } V_{0}=W_{0}, \quad s=t ; \\
& \text { (ii) } \operatorname{deg} \phi_{k}(x)=\operatorname{deg} \psi_{k}(x) \\
& \text { (iii) } V_{k-1}\left(\psi_{k}-\phi_{k}\right) \geq \mu_{k}=\nu_{k}
\end{aligned}
$$

$$
\begin{aligned}
& (k=1, \ldots, t) \\
& (k=1, \ldots, t) .
\end{aligned}
$$

The theorem is still true if either $s$ or $t$ is $+\infty$.

First prove the sufficiency of these conditions. Since (i) and (iii) make $V_{1}$ and $W_{1}$ identical, we can proceed by induction, assuming that $V_{k-1}=W_{k-1}$ is already established. Now compute the $V_{k}$ value of $\psi_{k}$. Because key polynomials have the leading coefficient unity, (ii) shows that the degree of $\phi_{k}$ exceeds that of $\psi_{k}-\phi_{k}$, so that $\psi_{k}$ has the expansion

$$
\psi_{k}=\phi_{k}+\left(\psi_{k}-\phi_{k}\right)
$$

in powers of $\phi_{k}$. The definition of $V_{k}$ and (iii) prove

$$
V_{k}\left(\psi_{i}\right)=\min \left\{\mu_{k}, V_{k-1}\left(\psi_{k}-\phi_{k}\right)\right\}=\mu_{k}=\nu_{k} .
$$

The $V_{k}$ value of any polynomial $f(x)$ can now be estimated from the expansion of $f(x)$ in $\psi_{k}$, for the triangle axiom for $V_{k}$ gives

$$
\begin{aligned}
V_{k}\left(\sum_{j=0}^{n} f_{j} \psi_{k}^{j}\right) \geq \min _{j}\left\{V_{k-1}\left(f_{j}\right)+j V_{k}\left(\psi_{k}\right)\right\} \\
=\min _{j}\left\{V_{k-1}\left(f_{j}\right)+j \nu_{k}\right\}=W_{k}(f),
\end{aligned}
$$

because of the definition of $W_{k}$. Thus $V_{k}(f) \geq W_{k}(f)$, while the inverse inequality is similarly proved. Hence $V_{k}=W_{k}$, and the induction is complete.

The necessity of the conditions depends chiefly on the invariance of the values assigned the key polynomials (Theorem 6.4). The assumption $V_{s}=W_{t}$ shows 
that $V_{0}=W_{0}$ and $\mu_{1}=\nu_{1}$. Hence (ii) and (iii) hold for $k=1$. We prove them by induction on $k$. If they hold through $k-1$, then the sufficiency proof shows $V_{k-1}=W_{k-1}$. By Theorem 5.1, deg $\phi_{k}$ can be characterized as the smallest degree of any polynomial $a(x)$ with the property that $V_{s}(a)>V_{k-1}(a)$. Since $V_{s}$ and $W_{t}$ are equal, $\operatorname{deg} \psi_{k}$ can be characterized by the same statement, so that

$$
\operatorname{deg} \phi_{k}(x)=\operatorname{deg} \psi_{k}(x) .
$$

The monotonic assumption on $\left\{\operatorname{deg} \phi_{k}\right\}$ then shows $V_{s}\left(\psi_{k}\right)=V_{k}\left(\psi_{k}\right)$. Hence, because of the invariance in $W$ of the value assigned to $\psi_{k}$,

$$
\nu_{k}=W_{t}\left(\psi_{k}\right)=V_{s}\left(\psi_{k}\right)=V_{k}\left(\phi_{k}+\left(\psi_{k}-\phi_{k}\right)\right) .
$$

As before, (6) is an expansion in powers of $\phi_{k}$, so that this equation becomes

$$
\nu_{k}=\min \left\{\mu_{k}, V_{k-1}\left(\psi_{k}-\phi_{k}\right)\right\} .
$$

Combining this with the symmetric conclusion (using $V_{k-1}=W_{k-1}$ )

$$
\mu_{k}=\min \left\{\nu_{k}, V_{k-1}\left(\psi_{k}-\phi_{k}\right)\right\} .
$$

we obtain (iii) for index $k$. With (7) this completes the induction. The condition $s=t$ results, even in the case $s=t=\infty$.

16. Normal forms for values. The results of the previous section do not apply to constant degree limit values, nor do they yield unique normal forms. Both these goals can be reached in the discrete case by using key polynomials from which all unnecessary high-valued terms have been dropped.

In the expansion of any $f(x)$ in a value $V_{k}$, the coefficient $f_{i}(x)$ of any power of $\phi_{k}$ can itself be expanded in powers of $\phi_{k-1}$. Since the degree of $f_{i}(x)$ is limited, the highest power of $\phi_{k-1}$ occurring is less than $n_{k} / n_{k-1}$, where $n_{i}$ has the meaning

$$
n_{i}=\operatorname{deg} \phi_{i}(x)
$$$$
(i=1, \ldots, k) .
$$

By an inductive process of this sort one can prove

Theorem 16.1. In any $V_{k}$ every polynomial $f(x)$ can be expanded as a polynomial in the key polynomials with constant coefficients,

$$
f(x)=\sum_{j} a_{j} \phi_{1}^{m_{1 j}} \phi_{2}^{m_{2 j}} \cdots \phi_{k}^{m_{k j}},
$$

where the exponents $m_{i j}$ are limited as follows

$$
m_{i j}<n_{i+1} / n_{i}
$$$$
\text { (all } j ; i=1,2, \ldots, k-1) .
$$

The value of $f(x)$, when computed from the definition, is

$$
V_{k}(f(x))=\min _{j}\left\{V_{k}\left(a_{j} \phi_{1}^{m_{1 j}} \cdots \phi_{k}^{m_{k j}}\right)\right\} .
$$

For a $p$-adic value, every number is equivalent to one of the numbers $c p^{m}, c=0$, $1, \ldots, p-1$. For any value $V_{0}$ of a field $K$ we can similarly (axiom of choice) pick from each class of equivalent elements a single representative element; in particular, we can make 1 one of the representatives. Given fixed representatives of this sort for each $V_{0}$, we say that a polynomial $f(x)$ is homogeneous in a value $V_{k}$ derived from $V_{0}$ if in the expansion (2) of $f(x)$ all the coefficients $a_{j}$ are representatives in $V_{0}$ and all the terms have the same minimum value $V_{k}(f(x))$.

Lemma 16.2. If $f(x)$ is a polynomial then $f(x) \approx_{V_{k}} h(x)$ for a unique homogeneous polynomial $h(x)$. This $h(x)$ is called the "homogeneous part" of $f(x)$.

Proof. Given $f(x)$, we find $h(x)$ by altering coefficients and dropping out terms in the expansion (2) for $f$. Were $f(x)$ also equivalent to a homogeneous $g(x)$, then all terms in the expansions of both $h(x)$ and $g(x)$ would have the same value $V_{k}(h)$, while $h-g$ would have a larger value. Thus corresponding coefficients are equivalent and therefore equal.

An inductive or limit value $V_{k}=\left[V_{0}, V_{i}\left(\phi_{i}\right)=\mu_{i}\right]$ may be called homogeneous if every key polynomial $\phi_{i}(x)$ is homogeneous in $V_{i-1}(i=2, \ldots, k)$. We will prove

Theorem 16.3. Any inductive or limit-value constructed from a discrete value $V_{0}$ of $K$ is equal to a homogeneous inductive or limit-value.

We have to prove that, if $U=\left[V_{k}, U(\phi)=\mu\right]$ is an augmented value over a homogeneous value $V_{k}$, then $U$ itself is equal to a homogeneous inductive value. This is done by introducing successive homogeneous parts of $\phi$ as new keys. First use $\psi_{1}(x)$, the homogeneous part of $\phi$ in $V_{k}$. By Lemma 16.2

$$
\psi_{1}(x) \approx_{V_{k}} \phi(x), \quad \operatorname{deg} \psi_{1}(x)=\operatorname{deg} \phi(x) .
$$

It follows that $\psi_{1}(x)$ is a key polynomial over $V_{k}$. Setting

$$
\nu_{1}=V_{k}\left(\phi(x)-\psi_{1}(x)\right)>V_{k}\left(\psi_{1}(x)\right),
$$

we can construct a homogeneous value $W_{1}=\left[V_{k}, W_{1}\left(\psi_{1}\right)=\nu_{1}\right]$. If $\mu \leq \nu_{1}$, then the sufficiency proof of Theorem 15.3 shows $U=W_{1}$. Otherwise $\mu>\nu_{1}$, and Corollary 13.2 proves $W^{\prime}=\left[W_{1}, W^{\prime}(\phi)=\mu\right]$ an inductive value, which by Lemma 15.1 is equal to $U$. We repeat the above argument, constructing a $W_{2}$ from $\psi_{2}(x)$, the principal part of $\phi$ in $W_{1}$. This gives a sequence of homogeneous inductive values,

$$
W_{1}=\left[V_{k}, W_{1}\left(\psi_{1}\right)=\nu_{1}, W_{2}\left(\psi_{2}\right)=\nu_{2}, \ldots, W_{t}\left(\psi_{t}\right)=\nu_{t}\right] \quad(i=1,2, \ldots) .
$$

The degrees of the $\psi(x)$ are all identical by (5), so that Lemma 6.3 proves that

$$
\nu_{1}<\nu_{2}<\nu_{3}<\cdots
$$


and that each $\nu_{i}$ is in the value-group $\Gamma_{k}$ of $V_{k}$. By hypothesis and Theorem 6.7, this $\Gamma_{k}$ is discrete. Hence there is a smallest $t$ with $\nu_{t} \geq \mu$ in (6), and $U$ is equal to the homogeneous value $W_{t}$. The advantage of so representing every value in a homogeneous form lies in the following uniqueness theorem:

Theorem 16.4. Two homogeneous inductive or limit-values which are equal must be identical.

Proof. If the equal values are $V_{s}$ and $W_{t}$ as in $\S 15,(4)$ and (5), then the asserted identity means simply that

$$
\begin{aligned}
& V_{0}=W_{0}, \\
& s=t, \\
& \phi_{k}=\psi_{k}, \\
& \mu_{k}=\nu_{k} \\
& (k=1,2, \ldots, s) .
\end{aligned}
$$

The hypotheses readily give $V_{0}=W_{0}$ and (8) for $k=1$.

Suppose (8) true up to $k-1$ inclusive. Then $V_{k-1}=W_{k-1}$. We can assume $s>k-1$, whence also $t>k-1$. Then $\phi_{k}$ has the following invariant properties which refer only to $V_{k-1}=W_{k-1}$ and $V_{s}=W_{t}$ : $\phi_{k}$ is totally homogeneous in $V_{k-1}$, it has the first coefficient 1 and it has the minimum degree consistent with the property $V_{s}\left(\phi_{k}\right)>V_{k-1}\left(\phi_{k}\right)$. Furthermore $\psi_{k}$ has the same properties. But these properties uniquely determine $\phi_{k}$, for, since the difference $\phi_{k}-\psi_{k}$ is of degree less than $\phi_{k}$, its value is

$$
\begin{aligned}
V_{k-1}\left(\phi_{k}-\psi_{k}\right) & =V_{s}\left(\phi_{k}-\psi_{k}\right) \\
& \geq \min \left\{V_{s}\left(\phi_{k}\right), V_{s}\left(\psi_{k}\right)\right\}>\min \left\{V_{k-1}\left(\phi_{k}\right), V_{k-1}\left(\psi_{k}\right)\right\} .
\end{aligned}
$$

Hence by the triangle law $\phi_{k} \approx_{V_{k-1}} \psi_{k}$, so that by Lemma $16.2, \phi_{k}=\psi_{k}$. By Theorem 6.4, $\mu_{k}=\nu_{k}$, as in (8). The induction ends when $k$ reaches $s=t$, and the identity $V_{s} \equiv W_{t}$ is proved.

\section{A simple consequence is}

Corollary 16.5. If every value of $K$ is discrete, then no inductive value can ever equal a limit-value, and no limit-value of constant degree type (\$7) can equal a limit-value not of this type.

\section{BIBLIOGRAPHY}

Papers on irreducibility:

(1846) Th. Schönemann, Von denjenigen Moduln, welche Potenzen von Primzahlen sind, Journal für die Mathematik 32 93-105, §61.

(1850) G. Eisenstein, Über die Irreduztibilität und einige andere Eigenschaften der Gleichung, von welcher die Theilung der ganzen Lemniscate abhängt, Journal für die Mathematik 39166.

(1895) L. Königsberger, Über den Eisensteinschen Satz von der Irreduzibilität algebraischer Gleichungen, Journal für die Mathematik 115 53-78; especially (67) on p 69.

(1905) M. Bauer, Verallgemeinerung eines Satzes von Schönemann, Journal für die Mathematik 128 87-89.

(1906) G. Dumas, Sur quelques cas d'irréductibilité des polynômes à coefficients rationnels, Journal de Mathématiques Pures et Appliquées (6) 2 191-258.

(1907) O. Perron, Neue Kriterien für die Irreduzibilität algebraischer Gleichungen, Journal für die Mathematik 132304.

(1916) H. Blumberg, On the factorization of expressions of various types, Transactions of the American Mathematical Society 17 517-544.

(1923) J. Kürschák, Irreduzible Formen, Journal für die Mathematik 152 180-191.

(1923) Ø. Ore, Zur Theorie der Irreduzibilitätskriterien, Mathematische Zeitschrift $18278-$ 288.

(1924) Ø. Ore, Zur Theorie der Eisensteinischen Gleichungen, Mathematische Zeitschrift $20267-279$.

(1927) T. Rella, Ordnungsbestimmungen in Integritätsbereichen und Newtonische Polygone, Journal für die Mathematik 158 33-48.

Papers on absolute values:

(1912) J. Kürschák, Limesbildung und allgemeine Körpertheorie, Journal für die Mathematik 142 211-253.

(1917) A. Ostrowski, Über einige Lösingen der Funktionalgleichung $\phi(x) \cdot \phi(y)=\phi(x y)$, Acta Mathematica 41 217-284.

(1930) W. Krull, Idealtheorie in unendlichen algebraischen Zahlkörpern II, Mathematische Zeitschrift 31 527-557.

(1932) W. Krull, Allgemeine Bewertungstheorie, Journal für die Mathematik 167 161-196.

(1933) H. Hasse and F. K. Schmidt, Die Struktur diskret bewerter Körper, Journal für die Mathematik 170 4-63.

(1934) A. Ostrowski, Untersuchungen zur arithmetischen Theorie der Körper, Mathematische Zeitschrift 39 269-404.

(1935) S. MacLane, Abstract absolute values which give new irreducibility criteria, Proceedings of the National Academy of Sciences 21 272-274.

(1936) S. MacLane, A construction for absolute values in polynomial rings, Transactions of the American Mathematical Society 40(3) 363-395.

(1936) S. MacLane, A construction for prime ideals as absolute values of an algebraic field, Duke Mathematical Journal 2 492-510. 